\title{
The feasibility of exercise interventions delivered via telehealth for people affected by cancer: a rapid review of the literature.
}

\author{
MORRISON, K.S., PATERSON, C. and TOOHEY, K.
}




\section{The feasibility of exercise interventions delivered via Telehealth for people affected by cancer: A rapid review of the literature}

Kittani S. Morrison (BExPhysRehab, AEP) ${ }^{\mathrm{a}}$, Catherine Paterson (PhD, BA, MSc, PgCert LTA, FHEA, RAN) $)^{\text {a,b,c,de, }}$, Kellie Toohey* (PhD, MCEP, BSc, PG Cert Research, PG Cert Tertiary Ed, AEP) $)^{\mathrm{a}, \mathrm{b}}$

${ }^{a}$ Faculty of Health, University of Canberra, Bruce ACT, Australia

${ }^{b}$ Prehabilitation, Activity, Cancer, Exercise and Survivorship (PACES) Research Group,

University of Canberra, Bruce ACT, Australia

${ }^{c}$ School of Nursing, Midwifery and Public Health, University of Canberra, Bruce ACT,

Australia

${ }^{d}$ Canberra Health Services \& ACT Health, SYNERGY Nursing \& Midwifery Research

Centre, ACT Health Directorate Level 3, Building 6, Canberra Hospital, Australia

${ }^{e}$ Robert Gordon University, Aberdeen, Scotland, UK

*Corresponding Author at: Faculty of Health, University of Canberra, Canberra Specialist

Medical Centre, Level C Office 3, Bruce 2617, Canberra, Australia.

E-mail address: kellie.toohey@canberra.edu.au (K. Toohey).

Declarations of interest: none. 


\begin{abstract}
Background: The prevalence of exercise as an adjunct therapy to cancer treatments including chemotherapy, radiation therapy and surgery is growing rapidly and has been shown to improve health outcomes, treatment completion rates and quality of life in people affected by cancer. Given the complexity of delivering cancer services during coronavirus disease (COVID-19), many people who are undergoing cancer treatment are unable to access exercise services. This review aims to investigate: a) the feasibility of exercise telehealth interventions for individuals diagnosed with cancer; and b) the impact of exercise telehealth interventions for people affected by cancer on physical and psycho-social outcomes.
\end{abstract}

Methods/Data Sources: The literature search was conducted in four electronic databases (CINAHL, Cochrane, Medline, and Psych Info) from the $1^{\text {st }}$ January 2010 until $1^{\text {st }}$ May 2020. All peer-reviewed qualitative and quantitative studies were included irrespective of study design. Studies that investigated adults (aged $\geq 18$ years) with a diagnosis of any cancer, irrespective of treatment type, cancer stage or primary/secondary nature of disease were included.

Results: Twenty-nine studies (a total of 3698 participants across the included studies) were synthesised. Across the included studies the interventions were broadly classified into four main areas of telehealth, web-based, mobile apps, SMS messaging, and telephone interventions.

Conclusion: Participants across the studies showed good compliance, symptom relief and reported an overall positive experience using telehealth for exercise. There were no adverse events reported in these studies. Given the current COVID-19 pandemic more research is required to assess the feasibility of telehealth platforms such as Zoom, Skype, Microsoft Teams or FaceTime, and to determine the overall participant and exercise professional telehealth exercise delivery experience.

Implications for nursing practice: Telehealth uses telecommunications technology as a tool to deliver health care to populations with limited access to cancer care. Quality care of a person living with cancer requires multidisciplinary team-based care and telecommunications technology can support interprofessional care. This review has underscored that telecommunications is a critical tool in the delivery of cancer care to enable timely ongoing support for exercise interventions for those affected by cancer. It remains important for 
people affected by cancer to continue to engage in and maintain regular exercise under the guidance of qualified health professionals in keeping with evidence-based clinical guidelines.

Key words: Telehealth, telemedicine, technology, telecommunications, cancer, exercise, oncology, physical activity. 


\section{INTRODUCTION}

The prevalence of exercise as an adjunct therapy to cancer treatments including chemotherapy, radiation therapy and surgery is growing rapidly and has been shown to improve health outcomes, treatment completion rates and quality of life in people affected by cancer. ${ }^{1}$ Exercise interventions can improve circulation, reduce cancer-related fatigue, improve body composition and general health and well-being in people affected by cancer. ${ }^{1}$ Given the complexity of delivering cancer services during coronavirus disease (COVID-19), many people who are undergoing cancer treatment are unable to access exercise services. A number of healthcare organizations have adopted telehealth as a way to circumvent the disruption to service delivery, due to social distancing measures, quarantine, self-isolation and hospital visitor limitations. ${ }^{2}$ Telehealth is defined by the Australian Government Department of Health as a broad range of telecommunication techniques to delivering telemedicine, medical education and health education over a distance. ${ }^{3}$ Prior to the global COVID-19 pandemic, telehealth research was already rapidly growing in cancer, cardiac, neurological and rehabilitation fields, but not main stream delivery of cancer services. ${ }^{4,5}$ Individuals affected by cancer are already at higher risk when compared to the general healthy population of developing infections, often leading to potentially life threatening complications. ${ }^{6}$ Preliminary evidence suggests people affected by cancer are more likely to experience serious illness if they become infected with COVID-19. ${ }^{7}$ COVID-19 poses a significant risk to people affected by cancer, particularly specific cancers including those: undergoing active chemotherapy and radical radiotherapy for lung cancer; blood or bone marrow cancers such as leukaemia or myeloma (at any stage of disease); those receiving immunotherapy; antibody treatments for cancer; targeted cancer treatments which can affect the immune system, such as protein kinase inhibitors; individuals who have received a bone marrow or stem cell transplant in the last six 
months; or those taking immunosuppression drugs. ${ }^{8}$ People affected by cancer could be at elevated risk of severe COVID-19 disease ${ }^{7}$ and may experience disrupted and delayed delivery of cancer therapies and exercise appointments because of general disruption in routine cancer service during the course of the ongoing pandemic. It remains important for people affected by cancer to continue to engage in and maintain regular exercise under the guidance of qualified health professionals in keeping with evidence-based clinical guidelines. ${ }^{1}$ Clinicians working with people affected by cancer are seeking research and guidance for delivering safe and successful evidence based telehealth exercise interventions to individuals diagnosed with cancer. Therefore, this timely review aims to investigate:

a) The feasibility of exercise telehealth interventions for individuals diagnosed with cancer.

b) The impact of exercise telehealth interventions for people affected by cancer on physical and psycho-social outcomes.

\section{METHODS}

\section{Search strategy}

The literature search was conducted in four electronic databases (CINAHL, Cochrane, Medline and Psych Info) from the $1^{\text {st }}$ January 2010 until $1^{\text {st }}$ May 2020. Electronic searches began on the $4^{\text {th }}$ May 2020 and concluded on $1^{\text {st }}$ June 2020. Medical subject headings (MeSH) for 'telehealth and 'exercise' were used. Search terms included were (telehealth OR telemedicine) AND (exercise OR physical activity OR exercise intervention) AND (cancer OR oncology). These search terms were combined using Boolean logic 'AND' and were searched in 'All Fields' of each electronic database. Reference lists of full text articles and systematic reviews were reviewed to ensure no studies were overlooked. 


\section{Inclusion and exclusion criteria}

All peer-reviewed qualitative and quantitative studies were included irrespective of study design. Studies that investigated adults (aged $\geq 18$ years) with a diagnosis of any cancer, irrespective of treatment type, cancer stage or primary/secondary nature of disease were included. Telehealth interventions were defined as: an exercise counselling or intervention delivered via phone, video conferencing, web-based or smartphone applications by a qualified health professional. ${ }^{3}$ Exercise counselling interventions were also included if they targeted exercise engagement and behaviour change strategies. Commentaries, narrative reviews, conference abstracts, study protocols, systematic reviews and editorials were excluded. Studies that were published in a language other than English, involved animals or in vitro experiments were also excluded.

\section{Data management and selection procedure}

Articles were imported into a web-based data management platform (Covidence $^{\complement}$ 2020, Version 1517, Melbourne, Australia) for screening according the inclusion and exclusion criteria. All duplicated were removed. Abstracts and titles were screened by two reviewers (KM and KT), with any conflicts resolved by a third reviewer (CP). All full texts were screened by two reviewers (KM and CP), and any conflicts were resolved by a third reviewer (KT). This review followed a rapid review methodology. A rapid review was appropriate in this current international COVID-19 public health emergency ${ }^{9}$, to take stock of the existing evidence to provide timely solutions to enable the safe delivery of telehealth interventions in exercise in cancer care.

\section{Quality appraisal}

Study quality was assessed using the Mixed Methods Appraisal Tool (2018 Version). ${ }^{10}$ The Mixed Methods Appraisal Tool includes specific criteria for qualitative, quantitative and mixed 
methods studies, focusing on methodological quality. ${ }^{10}$ Each study included in the quality appraisal was evaluated by one reviewer $(\mathrm{KM})$, and a second reviewer assessed a random sample of ten studies (KT). Any discrepancies were discussed between the reviewers and resolved. Every study was assigned a score based on each question within the appraisal tool, with a rating of 0-2 adopted to indicate low risk of bias -2 , unclear risk of bias -1 and high risk of bias -0 .

\section{Data extraction}

Data extraction was completed by one reviewer $(\mathrm{KM})$ in a Microsoft ${ }^{\odot}$ Excel spreadsheet. Initial data extraction included information on author and year, purpose and context of study, sample size, study design, time points, data collection, participant demographic and clinical characteristics, outcome measures and losses and exclusions of participants. Further data extraction captured feasibility related to adherence, completion rates and patient reported outcomes. Quantitative and qualitative data related to intervention outcomes, and any barriers and facilitators were also extracted.

\section{Data synthesis}

Due to the heterogeneity in study outcomes and measures across the included studies this rapid review used a narrative synthesis and tabulation of individual research studies to generate broad findings and conclusions. Given the heterogeneity across the studies there were no usable data to perform a meta-analysis. Specifically, the narrative synthesis involved data reduction (sub-group classification based on levels of evidence and the review questions), narrative data comparison (iterative process of making comparisons and identifying relationships) and finally, drawing conclusions. ${ }^{11}$ 


\section{RESULTS}

\section{Search results and overview}

The initial search yielded 145 records after the removal of duplicates. Based on the screening of titles and abstracts, a further 85 records were excluded following the application of the inclusion and exclusion criteria. Sixty studies were read in full-text and thirty were excluded with reasons, see Figure 1. Twenty-nine publications were included, and noteworthy, two studies $^{12,14}$ reported their results over two separate articles. ${ }^{12-14}$ Of the 27 research studies included a range of study designs, namely: two mixed methods, nine non-randomised studies and eighteen randomised studies. No additional studies were identified from review of the included studies reference lists. An overview of studies included is summarised in Table $\mathbf{1}$.

\section{Characteristics of the study samples.}

A total of 3698 participants were included across the studies and sample sizes ranged from $\mathrm{n}=10$ to $\mathrm{n}=518$. The included research studies represented global literature and were conducted in the following countries: USA $(n=9)$, Netherlands $(n=6)$, South Korea $(n=5)$, Australia ( $(n=3)$, Spain $(n=2)$, Scotland $(n=1)$, Turkey $(n=1)$, Canada $(n=1)$ and France $(n=1)$. The studies were conducted in people affected by breast $(n=12)$, mixed tumour $(n=6)$, colorectal $(n=2)$, head and neck $(n=1)$, gliomas $(n=1)$, endometrial $(n=2)$, lung $(n=2)$, prostate $(n=1)$ and haematological cancers $(n=2)$. Breast cancer represented $62 \%$ of studies. ${ }^{12-28}$ The participants varied by tumour stage and treatment modality across all studies. The mean age of the study participants was 54 years. Noteworthy, the participants were biased in favour of females, White Caucasian and educated, which limits our understanding of the use of telehealth in exercise delivery in other patient groups. 


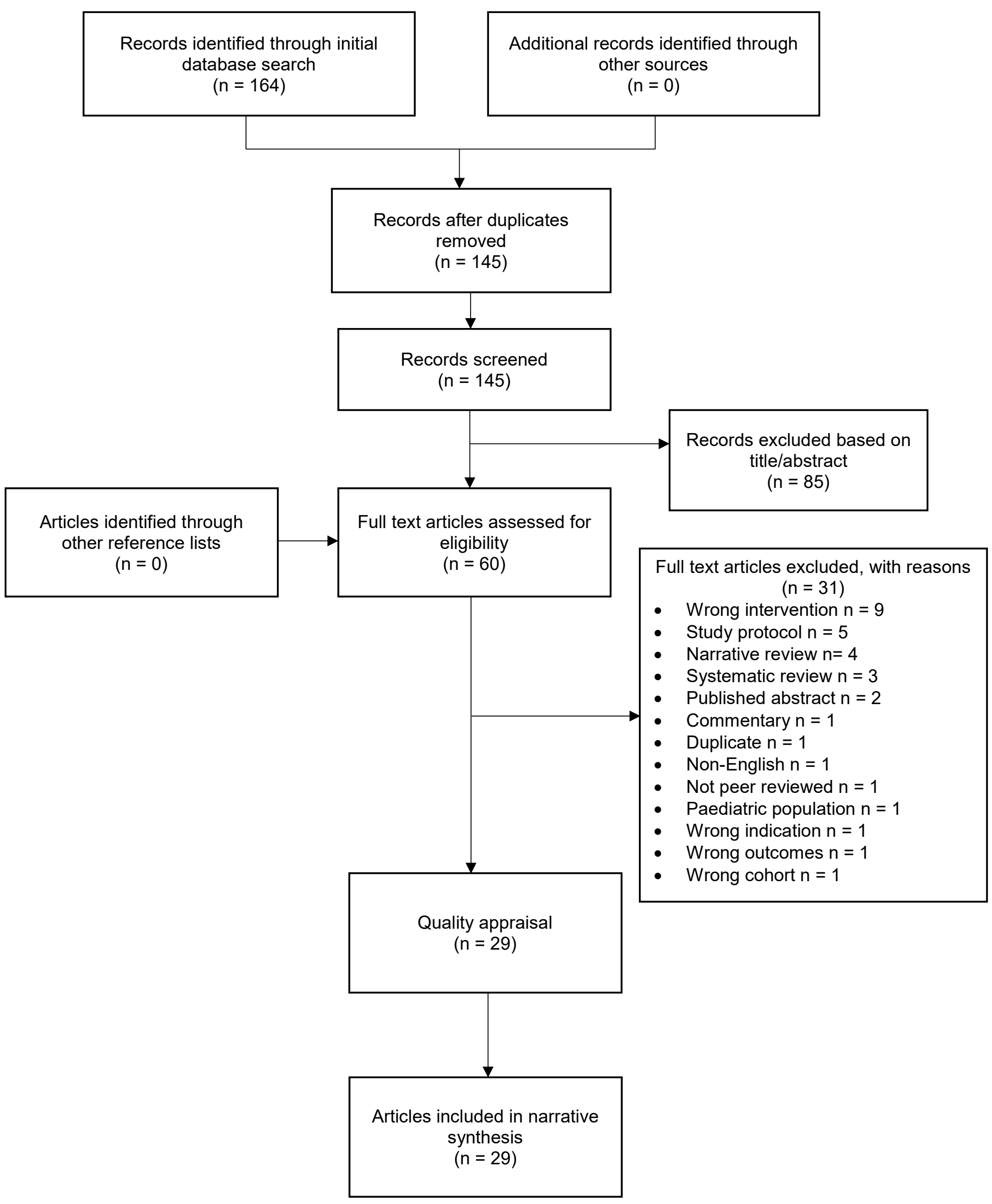

Figure 1. PRISMA flow diagram of the study selection, including literature search and reasons for exclusion. 
Table 1. Overview of the included articles $(\mathrm{N}=29)$.

\begin{tabular}{|c|c|c|c|c|c|}
\hline $\begin{array}{l}\text { Author, } \\
\text { Year and } \\
\text { Country }\end{array}$ & Purpose and Context & Methods & Participant Characteristics & $\begin{array}{l}\text { Outcome } \\
\text { Measures }\end{array}$ & $\begin{array}{l}\text { Losses and } \\
\text { Exclusions }\end{array}$ \\
\hline $\begin{array}{l}\text { Badger et } \\
\text { al., } 2017^{16} \\
\text { USA }\end{array}$ & $\begin{array}{l}\text { Purpose: To test the hypotheses that } \\
\text { telephone-delivered psychosocial } \\
\text { interventions decrease depression and } \\
\text { anxiety in women with breast cancer } \\
\text { and their partners. } \\
\text { Setting: Telehealth. } \\
\text { Recruitment: Two Universities and a } \\
\text { Health Science Centre. }\end{array}$ & $\begin{array}{l}\mathrm{n}=196 \text { (98 women } \\
\text { with breast cancer, } 98 \\
\text { partners) } \\
\text { Design: Three arm } \\
\text { randomised control. } \\
\text { Time points: } 3 \\
\text { Data collection: } \\
\text { Telephone interviews. }\end{array}$ & $\begin{array}{l}\text { Demographic: Mean age } 54 \text { y; } 100 \% \text { female; } 85 \% \\
\text { white; mean education level of high school or above; } \\
73 \% \text { married; } 64 \% \text { employed. } \\
\text { Clinical: breast cancer - } 33 \% \text { stage I, } 53 \% \text { stage II, } \\
14 \% \text { stage III; } 75 \% \text { receiving chemotherapy, } 54 \% \\
\text { radiation therapy, } 36 \% \text { hormone blocking therapy; } \\
25 \% \text { undergone a complete or radical mastectomy; all } \\
\text { primary episode of breast cancer. } \\
{ }^{*} \text { Partner characteristics not included. }\end{array}$ & $\begin{array}{l}\text { CES-D } \\
\text { Adapted Anxiety } \\
\text { Scale. }\end{array}$ & $\begin{array}{l}\text { Lost to follow } \\
\text { up: } n=16 \\
\text { Excluded } \\
\text { from } \\
\text { analysis: } \\
\mathrm{n}=16\end{array}$ \\
\hline $\begin{array}{l}\text { Bantum et } \\
\text { al., } 2014^{24} \\
\text { USA }\end{array}$ & $\begin{array}{l}\text { Purpose: To test the effectiveness of a } \\
\text { six-week web-based multiple health } \\
\text { behaviour change program for adult } \\
\text { survivors. } \\
\text { Setting: Telehealth. } \\
\text { Recruitment: One oncology centre and } \\
\text { one medical centre. }\end{array}$ & $\begin{array}{l}\mathrm{n}=352 \\
\text { Design: Two arm } \\
\text { randomised } \\
\text { controlled delayed } \\
\text { treatment. } \\
\text { Time points: } 2 \\
\text { Data collection: } \\
\text { Survey. }\end{array}$ & $\begin{array}{l}\text { Demographic: Mean age } 51 \text { y; } 92 \% \text { female; } 87 \% \\
\text { white; mean education level of } 16 \text { years. } \\
\text { Clinical: } 47 \% \text { breast cancer, } 40 \% \text { other, } 13 \% \text { female } \\
\text { gynaecological cancer; completion of primary } \\
\text { treatment four weeks prior; no other cancer diagnosis. }\end{array}$ & $\begin{array}{l}\text { BFI, WHIIRS, PHQ, } \\
\text { GLTEQ, BFFQ. }\end{array}$ & $\begin{array}{l}\text { Lost to follow } \\
\text { up: } n=49 \\
\text { Excluded } \\
\text { from } \\
\text { analysis: } \\
n=0\end{array}$ \\
\hline $\begin{array}{l}\text { Bruns et al., } \\
2019^{29} \\
\text { Netherlands }\end{array}$ & $\begin{array}{l}\text { Purpose: To assess the feasibility of a } \\
\text { home-based digital prehabilitation } \\
\text { program for frail elderly undergoing } \\
\text { surgery for colorectal cancer. } \\
\text { Setting: Telehealth. } \\
\text { Recruitment: Two hospitals. }\end{array}$ & $\begin{array}{l}\text { Sample size: } \mathrm{n}=14 \\
\text { Design: Pilot single } \\
\text { arm interventional. } \\
\text { Time points: } 2 \\
\text { Data collection: } \\
\text { Physical } \\
\text { assessments, self- } \\
\text { reported compliance, } \\
\text { and surveys. }\end{array}$ & $\begin{array}{l}\text { Demographic: Median age } 79 \text { y; } 64 \% \text { female. } \\
\text { Clinical: colorectal cancer - } 43 \% \text { stage I, } 43 \% \text { stage II, } \\
\text { 14\% stage II; surgery primary treatment. }\end{array}$ & $\begin{array}{l}\text { Fried Criteria, } \\
\text { Clinical Frailty } \\
\text { Scale, SPPB, } \\
\text { KATZ-ADL-6 Q, } \\
\text { MNA, MMSE, GDS, } \\
\text { EORTC QLQ. }\end{array}$ & $\begin{array}{l}\text { Lost to follow } \\
\text { up: } n=0 \\
\text { Excluded } \\
\text { from } \\
\text { analysis: } \\
n=0\end{array}$ \\
\hline $\begin{array}{l}\text { Cheong et } \\
\text { al., } 2018^{30} \\
\text { South Korea. }\end{array}$ & $\begin{array}{l}\text { Purpose: To evaluate the feasibility and } \\
\text { efficacy of comprehensive mobile } \\
\text { health care on quality of life and } \\
\text { physical performance of colorectal } \\
\text { cancer patients. } \\
\text { Setting: Telehealth. } \\
\text { Recruitment: One cancer clinic. }\end{array}$ & $\begin{array}{l}\text { Sample size: } \mathrm{n}=102 \\
\text { Design: Single arm } \\
\text { interventional. } \\
\text { Time points: } 2 \\
\text { Data collection: } \\
\text { Physical } \\
\text { assessments and } \\
\text { surveys. }\end{array}$ & $\begin{array}{l}\text { Demographic: Mean age } 58 \text { y; } 41 \% \text { female. } \\
\text { Clinical: Colorectal cancer diagnosis; all participants } \\
\text { currently undergoing chemotherapy. }\end{array}$ & $\begin{array}{l}\text { IPAQ-SF, PGA, } \\
\text { EORTC QLQ, grip } \\
\text { strength, } 30 \text { sec } \\
\text { STS, 2MWT, } \\
\text { NCCN-DT. }\end{array}$ & $\begin{array}{l}\text { Lost to follow } \\
\text { up: } n=27 \\
\text { Excluded } \\
\text { from } \\
\text { analysis: } \\
n=27\end{array}$ \\
\hline
\end{tabular}




\begin{tabular}{|c|c|c|c|c|c|}
\hline $\begin{array}{l}\text { Cheville et } \\
\text { al., } 2018^{31} \\
\text { USA }\end{array}$ & $\begin{array}{l}\text { Purpose: To evaluate the effectiveness } \\
\text { of collaborative telecare in preserving } \\
\text { function among patients with late stage } \\
\text { cancer and hematologic conditions. } \\
\text { Setting: Telehealth. } \\
\text { Recruitment: Several cancer and } \\
\text { medical centres. }\end{array}$ & $\begin{array}{l}\text { Sample size: } \mathrm{n}=516 \\
\text { Design: Three arm } \\
\text { randomised control. } \\
\text { Time points: } 3 \text { Data } \\
\text { collection: } \\
\text { Computerised } \\
\text { adaptive testing, } \\
\text { surveys and } \\
\text { telephone calls. }\end{array}$ & $\begin{array}{l}\text { Demographic: Mean age } 65 \text { y; } 50 \% \text { female, } 95 \% \\
\text { white; } 78 \% \text { married. } \\
\text { Clinical: All participants diagnosed with stage IIIC or } \\
\text { IV solid tumours, multiple myeloma, myelodysplastic } \\
\text { syndrome, or stage IIC or IV lymphoma. }\end{array}$ & $\begin{array}{l}\text { EQ-5D, FACIT } \\
\text { fatigue } \\
\text { questionnaire, } \\
\text { GLTEG, LASA } \\
\text { Scale for QOL, } \\
\text { CUR, BPI-SF, } \\
\text { Stanford Exercise } \\
\text { Behaviours } \\
\text { Questionnaire, } \\
\text { SOC-Q and Basic } \\
\text { Mobility. }\end{array}$ & $\begin{array}{l}\text { Lost to follow } \\
\text { up: } \mathrm{n}=75 \\
\text { Excluded } \\
\text { from } \\
\text { analysis: } \\
\mathrm{n}=\mathrm{NR}\end{array}$ \\
\hline $\begin{array}{l}\text { Chung et al., } \\
2019^{32} \\
\text { South Korea. }\end{array}$ & $\begin{array}{l}\text { Purpose: Investigate correlation } \\
\text { between physical activity data from } \\
\text { mobile devices and distress-related } \\
\text { questionnaire data from smartphone } \\
\text { apps in breast cancer survivors. } \\
\text { Setting: Telehealth. } \\
\text { Recruitment: One medical centre. }\end{array}$ & $\begin{array}{l}\text { Sample size: } \mathrm{n}=160 \\
\text { Design: Prospective } \\
\text { observational. } \\
\text { Time points: } 3 \\
\text { Data collection: Fitbit } \\
\text { app, surveys, and } \\
\text { telephone calls. }\end{array}$ & $\begin{array}{l}\text { Demographic: Mean age } 45 \mathrm{y} ; 100 \% \text { female, } 70 \% \\
\text { educational attainment of college level or higher; } 86 \% \\
\text { married; } 48 \% \text { employed. } \\
\text { Clinical: breast cancer - } 14 \% \text { stage } 0,48 \% \text { stage I, } \\
24 \% \text { stage II, } 14 \% \text { stage III; previous treatment } \\
\text { included chemotherapy, antihormonal therapy, } \\
\text { radiation therapy, surgery, and targeted therapy. }\end{array}$ & $\begin{array}{l}\text { NCCN-DT, PHQ-9, } \\
\text { HRQOL, EQ5D. }\end{array}$ & $\begin{array}{l}\text { Lost to follow } \\
\text { up: } n=33 \\
\text { Excluded } \\
\text { from } \\
\text { analysis: } \\
n=N R\end{array}$ \\
\hline $\begin{array}{l}\text { Chung et al., } \\
2020^{17} \\
\text { South Korea. }\end{array}$ & $\begin{array}{l}\text { Purpose: To investigate the effects of a } \\
\text { mobile app community program on } \\
\text { enhancing physical activity in breast } \\
\text { cancer survivors. } \\
\text { Setting: Telehealth. } \\
\text { Recruitment: One medical centre. }\end{array}$ & $\begin{array}{l}\text { Sample size: } \mathrm{n}=64 \\
\text { Design: Two arm } \\
\text { non-randomised, } \\
\text { prospective, } \\
\text { interventional study. } \\
\text { Time points: } 2 \text { Data } \\
\text { collection: Survey and } \\
\text { App data. }\end{array}$ & $\begin{array}{l}\text { Demographic: Mean age } 44 \text { y; } 100 \% \text { female; } 72 \% \\
\text { educational attainment of bachelor's degree; } 83 \% \\
\text { married; } 36 \% \text { employed. } \\
\text { Clinical: All participants diagnosed with breast cancer; } \\
\text { previous treatment included chemotherapy and anti- } \\
\text { hormonal therapy. }\end{array}$ & $\begin{array}{l}\text { NCCN-DT, daily } \\
\text { step count. }\end{array}$ & $\begin{array}{l}\text { Lost to follow } \\
\text { up: } n=27 \\
\text { Excluded } \\
\text { from } \\
\text { analysis: } \\
n=N R\end{array}$ \\
\hline $\begin{array}{l}\text { Cnossen et } \\
\text { al., } 2014^{33} \\
\text { Netherlands }\end{array}$ & $\begin{array}{l}\text { Purpose: To investigate the feasibility of } \\
\text { a self-help exercise program (Head } \\
\text { Matters) during treatment in head and } \\
\text { neck cancer patients. } \\
\text { Setting: Telehealth. } \\
\text { Recruitment: One university medical } \\
\text { centre. }\end{array}$ & $\begin{array}{l}\text { Sample size: } \mathrm{n}=34 \\
\text { Design: Mixed } \\
\text { methods. } \\
\text { Time points: } 2 \\
\text { Data collection: Self- } \\
\text { report diaries and } \\
\text { telephone interviews. }\end{array}$ & $\begin{array}{l}\text { Demographic: Mean age } 60 \text { y; } 24 \% \text { female. } \\
\text { Clinical: Stage I-IV head or neck cancer diagnosis; } \\
33 \% \text { radiation therapy, } 33 \% \text { chemotherapy and } 33 \% \\
\text { surgery. }\end{array}$ & $\begin{array}{l}\text { Adherence rates, } \\
\text { uptake percentage, } \\
\text { participant barriers } \\
\text { and facilitators to } \\
\text { exercise. }\end{array}$ & $\begin{array}{l}\text { Lost to follow } \\
\text { up: } n=1 \\
\text { Excluded } \\
\text { from } \\
\text { analysis: } \\
n=N R\end{array}$ \\
\hline $\begin{array}{l}\text { Eakin et al., } \\
2012^{18} \\
\text { Australia. }\end{array}$ & $\begin{array}{l}\text { Purpose: To evaluate the feasibility and } \\
\text { effectiveness of a telephone delivered } \\
\text { mixed exercise intervention for rural } \\
\text { women with breast cancer. }\end{array}$ & $\begin{array}{l}\text { Sample size: } \mathrm{n}=143 \\
\text { Design: Two arm } \\
\text { randomised control. } \\
\text { Time points: } 3\end{array}$ & $\begin{array}{l}\text { Demographic: Mean age } 53 \text { y; } 100 \% \text { female; } 47 \% \\
\text { educational attainment Year } 12 \text { or above; } 33 \% \\
\text { employed. }\end{array}$ & $\begin{array}{l}\text { Feasibility } \\
\text { indicators, AAS, } \\
\text { FACT-B+4, FACIT, }\end{array}$ & $\begin{array}{l}\text { Lost to follow } \\
\text { up: } n=6\end{array}$ \\
\hline
\end{tabular}




\begin{tabular}{|c|c|c|c|c|c|}
\hline & $\begin{array}{l}\text { Setting: Telehealth. } \\
\text { Recruitment: Eight rural hospitals. }\end{array}$ & $\begin{array}{l}\text { Data collection: } \\
\text { Exercise tracker, } \\
\text { surveys and } \\
\text { telephone interviews }\end{array}$ & $\begin{array}{l}\text { Clinical: breast cancer - } 40 \% \text { stage } 0 / I, 60 \% \text { stage II+; } \\
\text { treatment included chemotherapy, radiation therapy, } \\
\text { hormone therapy, Herceptin, and surgery. }\end{array}$ & $\begin{array}{l}\text { CHAMPS, STAI-Sf, } \\
\text { DASH-Q. }\end{array}$ & $\begin{array}{l}\text { Excluded } \\
\text { from } \\
\text { analysis: } \\
\mathrm{n}=6\end{array}$ \\
\hline $\begin{array}{l}\text { Frensham et } \\
\text { al., } 2018^{19} \\
\text { Australia. }\end{array}$ & $\begin{array}{l}\text { Purpose: To investigate the } \\
\text { effectiveness of an online tool } \\
\text { (STRIDE) designed to improve physical } \\
\text { activity among cancer survivors. } \\
\text { Setting: Telehealth. } \\
\text { Recruitment: One university. }\end{array}$ & $\begin{array}{l}\text { Sample size: } \mathrm{n}=102 \\
\text { Design: Two arm } \\
\text { randomised trial. } \\
\text { Time points: } 3 \text { Data } \\
\text { collection: } \\
\text { Pedometer, physical } \\
\text { assessments and } \\
\text { surveys. }\end{array}$ & $\begin{array}{l}\text { Demographic: Mean age } 66 \text { y; } 52 \% \text { female; } 96 \% \\
\text { white; } 97 \% \text { educational attainment of high school or } \\
\text { above; } 79 \% \text { married. } \\
\text { Clinical: } 42 \% \text { breast cancer, } 26 \% \text { other, } 22 \% \text { prostate } \\
\text { cancer; } 10 \% \text { colorectal; no participants undergoing } \\
\text { active treatment. }\end{array}$ & $\begin{array}{l}\text { BP, BMI, waist girth, } \\
6 \mathrm{MWT}, \text { SF-36, visits } \\
\text { to STRIDE website. }\end{array}$ & $\begin{array}{l}\text { Lost to follow } \\
\text { up: } n=19 \\
\text { Excluded } \\
\text { from } \\
\text { analysis: } \\
\mathrm{n}=11\end{array}$ \\
\hline $\begin{array}{l}\text { Galiano- } \\
\text { Castillo et } \\
\text { al., } 2017^{12} \\
\text { Spain }\end{array}$ & $\begin{array}{l}\text { Purpose: To determine if an Internet- } \\
\text { based tailored exercise intervention } \\
\text { leads to greater improvements in } \\
\text { functional capacity and cognition than } \\
\text { usual care in breast cancer survivors. } \\
\text { Setting: Telehealth. } \\
\text { Recruitment: One hospital. }\end{array}$ & $\begin{array}{l}\text { Sample size: } \mathrm{n}=81 \\
\text { Design: Two arm } \\
\text { randomised control. } \\
\text { Time points: } 3 \\
\text { Data collection: } \\
\text { Physical } \\
\text { assessments. }\end{array}$ & $\begin{array}{l}\text { Demographic: Mean age } 48 \text { y; } 100 \% \text { female; basic } \\
\text { educational level } 46 \% ; 69 \% \text { married; } 27 \% \text { employed. } \\
\text { Clinical: breast cancer - } 35 \% \text { stage I, } 52 \% \text { stage II, } \\
13 \% \text { stage IIIA; } 90 \% \text { received both radiation therapy } \\
\text { and chemotherapy. }\end{array}$ & 6MWT, ACT, TMT. & $\begin{array}{l}\text { Lost to follow } \\
\text { up: } n=5 \\
\text { Excluded } \\
\text { from } \\
\text { analysis: } \\
n=5\end{array}$ \\
\hline $\begin{array}{l}\text { Galiano- } \\
\text { Castillo et } \\
\text { al., } 2016^{13} \\
\text { Spain. }\end{array}$ & $\begin{array}{l}\text { Purpose: To investigate the } \\
\text { effectiveness of a telehealth system for } \\
\text { improving adverse effects in breast } \\
\text { cancer survivors. } \\
\text { Setting: Telehealth. } \\
\text { Recruitment: One hospital. } \\
\text { Country: }\end{array}$ & $\begin{array}{l}\text { Sample size: } \mathrm{n}=81 \\
\text { Design: Two arm } \\
\text { randomised control. } \\
\text { Time points: } 2 \text { Data } \\
\text { collection: Physical } \\
\text { assessments and } \\
\text { surveys. }\end{array}$ & $\begin{array}{l}\text { Demographic: Mean age } 48 \text { y; } 100 \% \text { female; basic } \\
\text { educational level } 46 \% ; 69 \% \text { married; } 27 \% \text { employed. } \\
\text { Clinical: breast cancer - } 35 \% \text { stage I, } 52 \% \text { stage II, } \\
13 \% \text { stage IIIA; } 90 \% \text { received both radiation therapy } \\
\text { and chemotherapy. }\end{array}$ & $\begin{array}{l}\text { EORTC QLQ-C30; } \\
\text { BPI; grip strength, } \\
\text { isometric abdominal } \\
\text { test, back } \\
\text { dynamometer, STS } \\
\text { test and PFS. }\end{array}$ & $\begin{array}{l}\text { Lost to follow } \\
\text { up: } n=5 \\
\text { Excluded } \\
\text { from } \\
\text { analysis: } \\
n=5\end{array}$ \\
\hline $\begin{array}{l}\text { Garrett et al., } \\
2013^{20} \\
\text { USA }\end{array}$ & $\begin{array}{l}\text { Purpose: To develop a feasibility study } \\
\text { of theory-driven telephone counselling } \\
\text { to enhance psychosocial and physical } \\
\text { wellbeing for cancer survivors. } \\
\text { Setting: Telehealth. } \\
\text { Recruitment: Two hospitals. }\end{array}$ & $\begin{array}{l}\text { Sample size: } \mathrm{n}=66 \\
\text { Design: Pilot single } \\
\text { arm interventional. } \\
\text { Time points: } 2 \text {. } \\
\text { Data collection: } \\
\text { Surveys. }\end{array}$ & $\begin{array}{l}\text { Demographic: Mean age } 60 \text { y; } 50 \% \text { female; } 89 \% \\
\text { white; } 100 \% \text { educational attainment high school level } \\
\text { or above; } 70 \% \text { married; } 46 \% \text { employed. } \\
\text { Clinical: } 39 \% \text { melanoma, } 24 \% \text { prostate cancer, } 22 \% \\
\text { breast cancer, } 15 \% \text { other; treatment types surgery, } \\
\text { chemotherapy and radiation therapy. }\end{array}$ & $\begin{array}{l}\text { IES, BFFQ and } \\
\text { physical activity } \\
\text { levels (mins/week). }\end{array}$ & $\begin{array}{l}\text { Lost to follow } \\
\text { up: } n=20 \\
\text { Excluded } \\
\text { from } \\
\text { analysis: } \\
n=20\end{array}$ \\
\hline $\begin{array}{l}\text { Gehring et } \\
\text { al., } 2018^{34} \\
\text { Netherlands }\end{array}$ & $\begin{array}{l}\text { Purpose: To investigate the feasibility of } \\
\text { a home-based exercise intervention for } \\
\text { patients with gliomas. } \\
\text { Setting: Telehealth. }\end{array}$ & $\begin{array}{l}\text { Sample size: } \mathrm{n}=34 \\
\text { Design: Two arm pilot } \\
\text { randomised control. } \\
\text { Time points: } 2 \text {. }\end{array}$ & $\begin{array}{l}\text { Demographic: Mean age } 48 \text { y; } 56 \% \text { female; } 96 \% \\
\text { educational attainment of middle or above. }\end{array}$ & $\begin{array}{l}\text { Patient emails, } \\
\text { IPAQ, VO } 2 \text { peak, } \\
\text { BMI, SF-36, letter }\end{array}$ & $\begin{array}{l}\text { Lost to follow } \\
\text { up: } n=6\end{array}$ \\
\hline
\end{tabular}




\begin{tabular}{|c|c|c|c|c|c|}
\hline & Recruitment: Three hospitals. & $\begin{array}{l}\text { Data collection: } \\
\text { Emails, physical } \\
\text { assessments and } \\
\text { surveys. }\end{array}$ & $\begin{array}{l}\text { Clinical: brain cancer - } 63 \% \text { grade II and } 37 \% \text { grade } \\
\text { III tumour; treatment types surgery, chemotherapy, } \\
\text { radiotherapy and oral medication. }\end{array}$ & $\begin{array}{l}\text { digit substitution } \\
\text { task. }\end{array}$ & $\begin{array}{l}\text { Excluded } \\
\text { from } \\
\text { analysis: } \\
\mathrm{n}=\mathrm{NR}\end{array}$ \\
\hline $\begin{array}{l}\text { Haggerty et } \\
\text { al., } 2017^{35} \\
\text { USA }\end{array}$ & $\begin{array}{l}\text { Purpose: To test the efficacy of } \\
\text { technology-based weight loss } \\
\text { interventions for endometrial cancer } \\
\text { survivors with obesity. } \\
\text { Setting: Telehealth. } \\
\text { Recruitment: Three medical centres. }\end{array}$ & $\begin{array}{l}\text { Sample size: } \mathrm{n}=41 \\
\text { Design: Three arm } \\
\text { randomised control. } \\
\text { Time points: } 2 . \\
\text { Data collection: } \\
\text { Physical } \\
\text { assessments and } \\
\text { surveys. }\end{array}$ & $\begin{array}{l}\text { Demographic: Mean age } 60 \text { y; } 100 \% \text { female; } 78 \% \\
\text { white. } \\
\text { Clinical: Endometrial cancer diagnosis, stage NR; no } \\
\text { current or future treatment planned. }\end{array}$ & $\begin{array}{l}\text { BMI, waist girth } \\
\text { (cm), SF-12, CRBI- } \\
\text { Q, FSFI-SS, IPAQ. }\end{array}$ & $\begin{array}{l}\text { Lost to follow } \\
\text { up: } n=9 \\
\text { Excluded } \\
\text { from } \\
\text { analysis: } \\
n=9\end{array}$ \\
\hline $\begin{array}{l}\text { Haggerty et } \\
\text { al., } 2016^{36} \\
\text { USA }\end{array}$ & $\begin{array}{l}\text { Purpose: To assess the feasibility of } \\
\text { two technology-based weight loss } \\
\text { interventions in obese women with } \\
\text { endometrial hyperplasia or cancer. } \\
\text { Setting: Telehealth. } \\
\text { Recruitment: Oncology centre. }\end{array}$ & $\begin{array}{l}\text { Sample size: } \mathrm{n}=20 \\
\text { Design: Two arm } \\
\text { randomised control. } \\
\text { Time points: } 2 \text {. } \\
\text { Data collection: } \\
\text { Biomarkers, physical } \\
\text { assessments and } \\
\text { surveys. }\end{array}$ & $\begin{array}{l}\text { Demographic: Mean age } 59 \text { y; } 100 \% \text { female; } 68 \% \\
\text { white. } \\
\text { Clinical: endometrial cancer - } 54 \% \text { stage IA, } 19 \% \\
\text { stage IB, } 5 \% \text { stage II, } 10 \% \text { stage IIIA, } 5 \% \text { stage IIIC, } \\
4 \% \text { stage IV, } 3 \% \text { unstaged. }\end{array}$ & $\begin{array}{l}\text { BMI, weight }(\mathrm{kg}) \\
\text { and inflammatory } \\
\text { biomarkers. }\end{array}$ & $\begin{array}{l}\text { Lost to follow } \\
\text { up: } n=N R \\
\text { Excluded } \\
\text { from } \\
\text { analysis: } \\
n=N R\end{array}$ \\
\hline $\begin{array}{l}\text { Ji et al., } \\
2019^{37} \\
\text { South Korea }\end{array}$ & $\begin{array}{l}\text { Purpose: To investigate the effects of a } \\
12 \text { week pulmonary rehabilitation } \\
\text { program using mobile patient health } \\
\text { data for individuals with lung cancer. } \\
\text { Setting: Telehealth. } \\
\text { Recruitment: One hospital. }\end{array}$ & $\begin{array}{l}\text { Sample size: } \mathrm{n}=64 \\
\text { Design: Two arm } \\
\text { randomised control. } \\
\text { Time points: } 3 \text {. } \\
\text { Data collection: } \\
\text { Physical } \\
\text { assessments and } \\
\text { surveys. }\end{array}$ & $\begin{array}{l}\text { Demographic: Mean age } 59 \text { y; } 29 \% \text { female. } \\
\text { Clinical: lung cancer }-31 \% \text { stage I, } 13 \% \text { stage II, } \\
20 \% \text { stage IIIA, } 1 \% \text { stage IIB, } 35 \% \text { stage IV. }\end{array}$ & $\begin{array}{l}\text { 6MWT, mMRC, EQ- } \\
\text { VAS, EQ-5D, PGA. }\end{array}$ & $\begin{array}{l}\text { Lost to follow } \\
\text { up: } n=21 \\
\text { Excluded } \\
\text { from } \\
\text { analysis: } \\
n=21\end{array}$ \\
\hline $\begin{array}{l}\text { Kanera et } \\
\text { al., } 2017^{14} \\
\text { Netherlands }\end{array}$ & $\begin{array}{l}\text { Purpose: To examine the long term } \\
\text { effects of a web-based program on } \\
\text { physical activity and vegetable } \\
\text { consumption in cancer survivors. } \\
\text { Setting: Telehealth. } \\
\text { Recruitment: Twenty one out-patient } \\
\text { clinics. }\end{array}$ & $\begin{array}{l}\text { Sample size: } \mathrm{n}=518 \\
\text { Design: Two arm } \\
\text { randomised control. } \\
\text { Time points: } 3 . \\
\text { Data collection: } \\
\text { Physical } \\
\text { assessments and } \\
\text { surveys. }\end{array}$ & $\begin{array}{l}\text { Demographic: Mean age } 56 \text { y; } 80 \% \text { female; } 62 \% \\
\text { educational attainment medium or above; } 51 \% \\
\text { employed. } \\
\text { Clinical: } 71 \% \text { breast cancer, } 29 \% \text { other; treatment } \\
\text { included chemotherapy, radiation therapy and } \\
\text { surgery. }\end{array}$ & $\begin{array}{l}\text { SQUASH, Dutch } \\
\text { Standard } \\
\text { Questionnaire on } \\
\text { Food Consumption, } \\
\text { BMI. }\end{array}$ & $\begin{array}{l}\text { Lost to follow } \\
\text { up: } n=56 \\
\text { Excluded } \\
\text { from } \\
\text { analysis: } \\
\mathrm{n}=10\end{array}$ \\
\hline $\begin{array}{l}\text { Kanera et } \\
\text { al., } 2016^{15} \\
\text { Netherlands }\end{array}$ & $\begin{array}{l}\text { Purpose: To assess the effects of a } \\
\text { web-based program on lifestyle } \\
\text { outcomes in cancer survivors who }\end{array}$ & $\begin{array}{l}\text { Sample size: } \mathrm{N}=518 \\
\text { Design: Two arm } \\
\text { randomised control. }\end{array}$ & $\begin{array}{l}\text { Demographic: Mean age } 56 \text { y; } 80 \% \text { female; } 62 \% \\
\text { educational attainment medium or above; } 51 \% \\
\text { employed. }\end{array}$ & $\begin{array}{l}\text { SQUASH, Dutch } \\
\text { Standard } \\
\text { Questionnaire on }\end{array}$ & $\begin{array}{l}\text { Lost to follow } \\
\text { up: } n=56\end{array}$ \\
\hline
\end{tabular}




\begin{tabular}{|c|c|c|c|c|c|}
\hline & $\begin{array}{l}\text { recently completed primary cancer } \\
\text { treatment. } \\
\text { Setting: Telehealth. } \\
\text { Recruitment: Twenty one hospitals. } \\
\text {. }\end{array}$ & $\begin{array}{l}\text { Time points: } 3 . \\
\text { Data collection: } \\
\text { Physical } \\
\text { assessments and } \\
\text { surveys. }\end{array}$ & $\begin{array}{l}\text { Clinical: } 71 \% \text { breast cancer, } 29 \% \text { other; treatment } \\
\text { included chemotherapy, radiation therapy and } \\
\text { surgery. }\end{array}$ & $\begin{array}{l}\text { Food Consumption, } \\
\text { BMl, smoking } \\
\text { status. }\end{array}$ & $\begin{array}{l}\text { Excluded } \\
\text { from } \\
\text { analysis: } \\
\mathrm{n}=10\end{array}$ \\
\hline $\begin{array}{l}\text { Ligibel et al., } \\
2010^{25} \\
\text { USA }\end{array}$ & $\begin{array}{l}\text { Purpose: To evaluate changes in } \\
\text { exercise behaviours in women } \\
\text { undergoing treatment for breast cancer } \\
\text { who participated in a telephone-based } \\
\text { physical activity intervention. } \\
\text { Setting: Telehealth. } \\
\text { Recruitment: One oncology clinic. }\end{array}$ & $\begin{array}{l}\text { Sample size: } \mathrm{n}=41 . \\
\text { Design: Pilot single } \\
\text { arm. } \\
\text { Time points: } 2 \text {. } \\
\text { Data collection: } \\
\text { Pedometer, physical } \\
\text { assessments and } \\
\text { surveys. }\end{array}$ & $\begin{array}{l}\text { Demographic: Mean age } 47 \text { y; } 100 \% \text { female. } \\
\text { Clinical: breast cancer - stage I } 27 \% \text {, stage II } 37 \% \text {, } \\
\text { stage III } 34 \% \text {, stage unknown } 2 \% \text {; treatment types } \\
\text { included surgery, chemotherapy and radiation } \\
\text { therapy. }\end{array}$ & $\begin{array}{l}\text { BMI, EORTC QLQ } \\
\text { C-30, Appearance } \\
\text { Orientation Scale, } \\
\text { modified } \mathrm{VO}_{2} \text { max, } \\
\text { minutes/week of } \\
\text { activity. }\end{array}$ & $\begin{array}{l}\text { Lost to follow } \\
\text { up: } n=7 \\
\text { Excluded } \\
\text { from } \\
\text { analysis: } \\
n=N R\end{array}$ \\
\hline $\begin{array}{l}\text { Mohamad et } \\
\text { al., } 2019^{38} \\
\text { Scotland }\end{array}$ & $\begin{array}{l}\text { Purpose: To investigate changes in a } \\
\text { diet and physical activity intervention on } \\
\text { body weight and quality of life in men } \\
\text { treated for prostate cancer. } \\
\text { Setting: Telehealth. } \\
\text { Recruitment: Cancer database. }\end{array}$ & $\begin{array}{l}\text { Sample size: } \mathrm{n}=62 . \\
\text { Design: Pilot two arm } \\
\text { randomised. } \\
\text { Time points: } 4 . \\
\text { Data collection: } \\
\text { Physical } \\
\text { assessments, survey } \\
\text { and website. }\end{array}$ & $\begin{array}{l}\text { Demographic: Mean age } 66 \text { y; } 0 \% \text { female. } \\
\text { Clinical: diagnosis of localised or locally advanced } \\
\text { prostate cancer within the last } 36 \text { months. }\end{array}$ & $\begin{array}{l}\text { Body weight, } \\
\text { EORTC QLQ-C30, } \\
\text { EORTC QLQ-PR25, } \\
\text { website use, }\end{array}$ & $\begin{array}{l}\text { Lost to follow } \\
\text { up: } n=28 \\
\text { Excluded } \\
\text { from } \\
\text { analysis: } \\
n=N R\end{array}$ \\
\hline $\begin{array}{l}\text { Nemli et al., } \\
2019^{26} \\
\text { Turkey }\end{array}$ & $\begin{array}{l}\text { Purpose: To determine the effects of } \\
\text { exercise training supported by } \\
\text { telephone calls on postoperative } \\
\text { physical activity and quality of life levels } \\
\text { of women with breast cancer. } \\
\text { Setting: Telehealth. } \\
\text { Recruitment: One university hospital. }\end{array}$ & $\begin{array}{l}\text { Sample size: } \mathrm{n}=62 \\
\text { Design: Two arm } \\
\text { randomised control. } \\
\text { Time points: } 2 . \\
\text { Data collection: } \\
\text { Physical } \\
\text { assessments, } \\
\text { surveys and } \\
\text { telephone interviews. }\end{array}$ & $\begin{array}{l}\text { Demographic: Mean age } 51 \mathrm{y} ; 100 \% \text { female. } \\
\text { Clinical: breast cancer diagnosis; currently } \\
\text { undergoing chemotherapy treatment; prior treatment } \\
\text { included surgery. }\end{array}$ & IPAQ, QOL-Q, BMI. & $\begin{array}{l}\text { Lost to follow } \\
\text { up: } n=N R \\
\text { Excluded } \\
\text { from } \\
\text { analysis: } \\
n=0\end{array}$ \\
\hline $\begin{array}{l}\text { Quintiliani et } \\
\text { al., } 2016^{21} \\
\text { USA }\end{array}$ & $\begin{array}{l}\text { Purpose: To evaluate a weight } \\
\text { management intervention via mobile } \\
\text { health for breast cancer survivors. } \\
\text { Setting: Telehealth. } \\
\text { Recruitment: One university. }\end{array}$ & $\begin{array}{l}\text { Sample size: } \mathrm{n}=10 \\
\text { Design: Single arm } \\
\text { pilot. } \\
\text { Time points: } 2 . \\
\text { Data collection: } \\
\text { Physical } \\
\text { assessments and } \\
\text { surveys. }\end{array}$ & $\begin{array}{l}\text { Demographic: Mean age } 59 \text { y; } 60 \% \text { white; } 50 \% \\
\text { university graduate or higher. } \\
\text { Clinical: breast cancer - stage } 0-160 \% \text {, stage II + } \\
40 \% \text {. }\end{array}$ & $\begin{array}{l}\text { BMI, Prime Screen, } \\
\text { BEVQ-15, IPAQ, } \\
\text { PSS, Loss of } \\
\text { Control over eating } \\
\text { Scale-Brief. }\end{array}$ & $\begin{array}{l}\text { Lost to follow } \\
\text { up: } n=0 \\
\text { Excluded } \\
\text { from } \\
\text { analysis: } \\
n=0\end{array}$ \\
\hline
\end{tabular}




\begin{tabular}{|c|c|c|c|c|c|}
\hline $\begin{array}{l}\text { Rocque et } \\
\text { al., } 2017^{22} \\
\text { USA }\end{array}$ & $\begin{array}{l}\text { Purpose: To assess the feasibility of a } \\
\text { telehealth pain and fatigue self- } \\
\text { management program among adult } \\
\text { cancer patients. } \\
\text { Setting: Telehealth. } \\
\text { Recruitment: One university. }\end{array}$ & $\begin{array}{l}\text { Sample size: } \mathrm{n}=40 \\
\text { Design: Non- } \\
\text { randomised pre-post } \\
\text { evaluation. } \\
\text { Time points: } 2 . \\
\text { Data collection: } \\
\text { Survey. }\end{array}$ & $\begin{array}{l}\text { Demographic: Mean age } 62 \text { y; } 80 \% \text { female; } 75 \% \\
\text { white, } 50 \% \text { married. } \\
\text { Clinical: } 30 \% \text { breast cancer, } 34 \% \text { other, } 17 \% \\
\text { hematologic cancer, } 12 \% \text { lung cancer, } 7 \% \text { colorectal } \\
\text { cancer. }\end{array}$ & $\begin{array}{l}\text { Study participation, } \\
\text { PAM, NCCN } \\
\text { distress } \\
\text { thermometer, pain, } \\
\text { fatigue, MDASI, SF- } \\
12 .\end{array}$ & $\begin{array}{l}\text { Lost to follow } \\
\text { up: } n=20 \\
\text { Excluded } \\
\text { from } \\
\text { analysis: } \\
n=N R\end{array}$ \\
\hline $\begin{array}{l}\text { Short et al., } \\
2017^{23} \\
\text { Australia }\end{array}$ & $\begin{array}{l}\text { Purpose: To investigate the impact of a } \\
\text { web-based intervention on physical } \\
\text { activity levels targeting breast cancer } \\
\text { survivors. } \\
\text { Setting: Telehealth. } \\
\text { Recruitment: Cancer support groups. }\end{array}$ & $\begin{array}{l}\text { Sample size: } \mathrm{n}=492 \\
\text { Design: Three arm } \\
\text { randomised } \\
\text { experimental. } \\
\text { Time points: } 3 . \\
\text { Data collection: } \\
\text { Physical } \\
\text { assessments, } \\
\text { surveys and website } \\
\text { data. }\end{array}$ & $\begin{array}{l}\text { Demographic: Mean age } 55 \text { y; } 100 \% \text { female; } 48 \% \\
\text { university level of education; } 74 \% \text { married; } 48 \% \\
\text { employed. } \\
\text { Clinical: breast cancer - } 2 \% \text { stage } 0,22 \% \text { stage I, } \\
31 \% \text { stage II, } 21 \% \text { stage III, } 4 \% \text { stage IV, unsure } \\
20 \% \text {. }\end{array}$ & $\begin{array}{l}\text { Website } \\
\text { acceptability, } \\
\text { website usability, } \\
\text { website usage, } \\
\text { QLTEQ, BMI. }\end{array}$ & $\begin{array}{l}\text { Lost to follow } \\
\text { up: } n=439 \\
\text { Excluded } \\
\text { from } \\
\text { analysis: } \\
n=0\end{array}$ \\
\hline $\begin{array}{l}\text { Timmerman } \\
\text { et al., } 2017^{39} \\
\text { Netherlands }\end{array}$ & $\begin{array}{l}\text { Purpose: To evaluate the feasibility of a } \\
\text { telehealth care application for operable } \\
\text { lung cancer. } \\
\text { Setting: Telehealth. } \\
\text { Recruitment: One cancer institute. } \\
\text {. }\end{array}$ & $\begin{array}{l}\text { Sample size: } \mathrm{n}=17 \\
\text { Design: Single arm } \\
\text { mixed methods } \\
\text { feasibility. } \\
\text { Time points: } 4 . \\
\text { Data collection: } \\
\text { Focus groups, } \\
\text { survey, and website } \\
\text { data. }\end{array}$ & $\begin{array}{l}\text { Demographic: Mean age } 59 \text { y; } 47 \% \text { female. } \\
\text { Clinical: diagnosis of primary non-small lung cancer; } \\
\text { scheduled for curative lung resection. }\end{array}$ & $\begin{array}{l}\text { Use and } \\
\text { acceptability, } \\
\text { participant } \\
\text { satisfaction, } \\
\text { symptom, and } \\
\text { physical activity } \\
\text { monitoring, }\end{array}$ & $\begin{array}{l}\text { Lost to follow } \\
\text { up: } n=4 \\
\text { Excluded } \\
\text { from } \\
\text { analysis: } \\
n=N R\end{array}$ \\
\hline $\begin{array}{l}\text { Uhm et al., } \\
2017^{27} \\
\text { South Korea }\end{array}$ & $\begin{array}{l}\text { Purpose: To investigate and compare } \\
\text { the effects of mobile health and } \\
\text { pedometers with conventional exercise } \\
\text { on physical activity and quality of life in } \\
\text { breast cancer patients. } \\
\text { Setting: Telehealth. } \\
\text { Recruitment: Three hospitals. } \\
\text { Country. }\end{array}$ & $\begin{array}{l}\text { Sample size: } \mathrm{n}=356 \\
\text { Design: Two arm } \\
\text { randomised trial. } \\
\text { Time points: } 3 \text {. } \\
\text { Data collection: } \\
\text { Physical } \\
\text { assessments and } \\
\text { surveys. }\end{array}$ & $\begin{array}{l}\text { Demographic: Mean age } 50 \text { y; } 100 \% \text { female. } \\
\text { Clinical: breast cancer - stage } 06 \% \text {, stage IA 33\%, } \\
\text { stage IB 3\%, stage IIA } 22 \% \text {, stage IIB } 10 \% \text {, stage IIA } \\
8 \% \text {, stage IIBC } 1 \% \text {, stage IIC 3\%, stage IV } 1 \%, 13 \% \\
\text { unknown; treatment included surgery, chemotherapy, } \\
\text { radiotherapy, hormonal therapy and targeted drug } \\
\text { therapy. }\end{array}$ & $\begin{array}{l}\text { IPAQ-SF, EORTC- } \\
\text { QOLQ-C30, } \\
\text { EORTC-QOLQ- } \\
\text { BR23, BMI, BP, PR, } \\
\text { arm circumferences, } \\
\text { handgrip strength, } \\
30 \text { second STS, } \\
\text { 2MWT. }\end{array}$ & $\begin{array}{l}\text { Lost to follow } \\
\text { up: } n=17 \\
\text { Excluded } \\
\text { from } \\
\text { analysis: } \\
n=17\end{array}$ \\
\hline $\begin{array}{l}\text { Vallerand et } \\
\text { al., } 2019^{40} \\
\text { Canada }\end{array}$ & $\begin{array}{l}\text { Purpose: To examine changes in social } \\
\text { cognitive variables from a telephone } \\
\text { counselling exercise intervention in } \\
\text { hematologic cancer survivors. } \\
\text { Setting: Telehealth. }\end{array}$ & $\begin{array}{l}\text { Sample size: } \mathrm{n}=51 \\
\text { Design: Two arm } \\
\text { randomised control. } \\
\text { Time points: } 2 \text {. }\end{array}$ & $\begin{array}{l}\text { Demographic: Mean age } 53 \text { y; } 61 \% \text { female; } 63 \% \\
\text { completed university; } 76 \% \text { married; } 80 \% \text { employed. } \\
\text { Clinical: } 43 \% \text { Hodgkin Iymphoma, } 37 \% \text { Leukemia, } \\
20 \% \text { non-Hodgkin lymphoma; treatment included }\end{array}$ & $\begin{array}{l}\text { Motivational } \\
\text { processes, } \\
\text { behavioural } \\
\text { regulations, }\end{array}$ & $\begin{array}{l}\text { Lost to follow } \\
\text { up: } n=0 \\
\text { Excluded } \\
\text { from } \\
\text { analysis: }\end{array}$ \\
\hline
\end{tabular}




\begin{tabular}{|c|c|c|c|c|c|}
\hline & $\begin{array}{l}\text { Recruitment: Two Universities. } \\
\text { Country. }\end{array}$ & $\begin{array}{l}\text { Data collection: } \\
\text { Surveys. }\end{array}$ & $\begin{array}{l}\text { radiation therapy, chemotherapy, stem cell or marrow } \\
\text { transplant. }\end{array}$ & $\begin{array}{l}\text { reflexive processes, } \\
\text { GLTEQ, SF-36. }\end{array}$ & $\mathrm{n}=0$ \\
\hline $\begin{array}{l}\text { Villaron et } \\
\text { al., } 2018^{28} \\
\text { France }\end{array}$ & $\begin{array}{l}\text { Purpose: To identify the effects of } \\
\text { recommendations and telehealth on } \\
\text { physical activity levels, fatigue and } \\
\text { quality of life. } \\
\text { Setting: Telehealth. } \\
\text { Recruitment: One cancer centre. }\end{array}$ & $\begin{array}{l}\text { Sample size: } \mathrm{n}=60 \\
\text { Design: } \text { Randomised } \\
\text { pilot study. } \\
\text { Time points: } 9 . \\
\text { Data collection: } \\
\text { Surveys and } \\
\text { pedometer. }\end{array}$ & $\begin{array}{l}\text { Demographic: Mean age } 50 \text { y; } 72 \% \text { female. } \\
\text { Clinical: } 49 \% \text { breast cancer, } 12 \% \text { other, } 11 \% \\
\text { Hodgkin's lymphoma, } 9 \% \text { ovarian cancer, } 9 \% \\
\text { colorectal cancer. }\end{array}$ & $\begin{array}{l}\text { Pedometer steps, } \\
\text { MFI-20, EORTC- } \\
\text { QLQ-C30. }\end{array}$ & $\begin{array}{l}\text { Lost to follow } \\
\text { up: } n=17 \\
\text { Excluded } \\
\text { from } \\
\text { analysis: } \\
n=17\end{array}$ \\
\hline
\end{tabular}

CES-D: Centre for Epidemiological Studies Depression Scale; BFI: Brief Fatigue Inventory; WHIIRS: Women's Health Initiative Insomnia Rating Scale; PHQ: Patient Health Questionnaire; GLTEQ: Godin Leisure Time Exercise Questionnaire; BFFQ: Block Food Frequency Questionnaire; SPPB: Short Physical Performance Battery; KATZ-ADL-6 Q: KATZ Independence of Activities of Daily Living; MNA: Mini Nutritional Assessment; MMSE: Mini Mental State Exam; GDS: Geriatric Depression Scale; EORTC QLQ: European Organisation for Research and Treatment of Cancer Quality of Life Questionnaire; IPAQ-SF: International Physical Activity Questionnaire - Short Form; PGA: Patient Global Assessment ;STS: Sit-to-Stand; 2MWT: Two Minute Walk Test; NCCN-DT: National Comprehensive Cancer Network - Distress Thermometer; EQ-5D: Euroqol 5-Dimensions; FACIT: Functional Assessment of Chronic Illness Therapy-Fatigue Scale; LASA: Linear Analogue SelfAssessment; CUR: Care Utilization Report; BPI-SF: Brief Pain Inventory Short Form; SOC-Q: Stages of Change Questionnaire; PHQ-9: Patient Health Questionnaire-9; HRQOL: Health Related Quality of Life; AAS: Active Australia Survey; FACT-B+4: Functional Assessment of Cancer Therapy-Breast Questionnaire; CHAMPS: Community Healthy Activities Models Programs for Seniors; STAI-Sf: State Trait Anxiety Inventory; DASH-Q: Disability of Arm. Shoulder and Hand Questionnaire; BP: Blood Pressure; BMI: Body Mass Index; 6MWT: Six Minute Walk Test; SF-36: Short Form Healthy Survey; ACT: Auditory Consonant Trigrams; TMT: Trail Making Test; BPI: Brief Pain Inventory; PFS: Piper Fatigue Scale; IES: Impact of Event Scale; NR: Not Reported; SF-12: Physical Health Component; CRBI: Cancer-Related Body Image Questionnaire; FSFS-SS: Female Sexual Functioning Satisfaction - Subscale; mMRC: modified Medical Research Council; EQ-VAS: EuroQOL-visual analogue scale; SQUASH: Short Questionnaire to assess Health Enhancing Physical Activity; EORTC QLQ-PR25: European Organisation for Research and Treatment of Cancer Quality of Life Questionnaire - Prostate Cancer; QOL-Q: Quality of Life Questionnaire; BEVQ-15: 15-Item Beverage Questionnaire; PSS: Perceived Stress Scale; PAM: Patient Activation Measure; Quality of Life Questionnaire - Prostate Cancer, QOL-Q: Quality of Life Questionnaire; BEVQ-15: 15-ltem Beverage Questionnaire; PSS. Percelved Stress Scale; PAM. Patient Activalion Measure; MDASI: MD Anderson Symptom Inventory; EORTC-QOLQ-BR23: European Organisation for Research and Treatment of Cancer Quality of Life Questionnaire - Breast Cancer; PR: Pulse Rate; MFI-20: Modified Fatigue Index - 20. 


\section{Quality appraisal results}

The results of the quality appraisal are presented in Table 2. No articles scored a low risk of bias across all scoring domains. In the two mixed methods studies ${ }^{33,39}$ the qualitative component of both studies did not adhere to the quality criteria of this methodological approach. Specifically, the qualitative component lacked adequate description of the methods utilised $^{33,39}$. The non-randomised studies lacked complete outcome data due to participant dropout rates and as such the results from these studies are viewed with caution. There were several issues in the included randomised controlled studies worth a mention, 1) several studies $^{16,18,26,28,34-37,40}$ did not describe the randomisation approach, 2) the primary researcher was in some cases not blinded to the randomisation process ${ }^{16,18}$ and 3) in the majority of the randomised studies the outcome assessors were not blinded to the intervention administered. ${ }^{14-16,18,23,24,26,28,31,34-38,40}$

\section{Patient Experiences of Telehealth Exercise Interventions}

\section{Interventions}

Across the included studies the interventions were broadly classified into four main areas of telehealth, web-based, mobile apps, SMS messaging, and telephone interventions, see Table 3. The majority of the studies used a web-based platform to deliver the exercise intervention ${ }^{12-15,19,23,24,29,31,35-39}$ and in one study with the addition of pedometers. ${ }^{19}$ Elsewhere other studies use a variety of mobile apps ${ }^{17,18,27,32}$ with, or without pedometers/other wearables. Other approaches to exercise intervention included weekly SMS with pedometers. $^{28}$ The remainder of the studies used traditional approaches which included telephone counselling or email communication with healthcare 
professionals $^{16,18,20,25,26}$ and two studies ${ }^{21,36}$ used remote telephone counselling with Wi-Fi

tracking devices and SMS messaging. Interestingly, none of the included studies reported

using face-to-face telehealth platforms such as Zoom, Skype, Microsoft Teams or FaceTime.

Table 2. Results of the quality appraisal of articles

\begin{tabular}{|c|c|c|c|c|c|c|c|}
\hline \multirow{2}{*}{ Study type } & \multicolumn{7}{|c|}{ Item Number of Checklist } \\
\hline & 1 & 2 & 3 & 4 & 5 & 6 & 7 \\
\hline \multicolumn{8}{|c|}{ - $\quad$ Mixed Methods Studies ${ }^{a}$} \\
\hline Cnossen et al., $2014^{33}$ & 2 & 2 & 2 & 1 & 1 & 1 & 0 \\
\hline Timmerman et al., $2017^{39}$ & 2 & 2 & 2 & 1 & 1 & 1 & 0 \\
\hline \multicolumn{8}{|c|}{$\begin{array}{l}\text { a Mixed Methods Item number check list key } \\
1 \text { - Are there clear research questions? } \\
2 \text { - Does the collected data answer the research questions? } \\
3 \text { - Is there an adequate rationale for using a mixed methods design to address the research question? } \\
4 \text { - Are the different components of the study effectively integrated to answer the research question? } \\
5 \text { - Are the outputs of the integration of qualitative and quantitative components adequately interpreted? } \\
6 \text { - Are divergences and inconsistencies between quantitative and qualitative results adequately addressed? } \\
7 \text { - Do the different components of the study adhere to the quality criteria of each tradition of the methods involved? }\end{array}$} \\
\hline \multicolumn{8}{|c|}{ - $\quad$ Non-Randomised Studies ${ }^{c}$} \\
\hline Bruns et al., $2019^{29}$ & 2 & 2 & 2 & 2 & 2 & 1 & 2 \\
\hline Cheong et al., $2018^{30}$ & 2 & 2 & 2 & 2 & 0 & 1 & 2 \\
\hline Chung et al., $2019^{32}$ & 2 & 2 & 2 & 2 & 0 & 2 & 2 \\
\hline Chung et al., $2020^{17}$ & 2 & 2 & 2 & 2 & 0 & 2 & 2 \\
\hline Frensham et al., $2018^{19}$ & 2 & 2 & 2 & 2 & 2 & 2 & 1 \\
\hline Garret et al., $2013^{20}$ & 2 & 2 & 2 & 2 & 1 & 1 & 2 \\
\hline Ligibel et al., $2010^{25}$ & 2 & 2 & 2 & 2 & 0 & 2 & 2 \\
\hline Quintiliani et al., $2016^{21}$ & 2 & 2 & 2 & 2 & 2 & 1 & 2 \\
\hline Rocque et al., $2017^{22}$ & 2 & 2 & 2 & 2 & 0 & 1 & 2 \\
\hline \multicolumn{8}{|c|}{$\begin{array}{l}\text { 'Item number check list key } \\
1 \text { - Are there clear research questions? } \\
2 \text { - Does the collected data answer the research questions? } \\
3 \text { - Are the participants representative of the target population? } \\
4 \text { - Are measurements appropriate regarding the outcome and intervention? } \\
5 \text { - Are there complete outcome data? } \\
6 \text { - Are the confounders accounted for in the design and analysis? } \\
7 \text { - During the study period, is the intervention administered as intended? }\end{array}$} \\
\hline \multicolumn{8}{|c|}{ - Randomised Studies $^{d}$} \\
\hline Badger et al., $2017^{16}$ & 2 & 2 & 0 & 0 & 2 & 0 & 2 \\
\hline Bantum et al., $2014^{24}$ & 2 & 2 & 2 & 2 & 2 & 0 & 2 \\
\hline Cheville et al., $2018^{31}$ & 2 & 2 & 2 & 2 & 2 & 0 & 0 \\
\hline Eakin et al., $2012^{18}$ & 2 & 2 & 0 & 2 & 2 & 0 & 2 \\
\hline $\begin{array}{l}\text { Galliano-Castillo et al., } \\
2017^{12}\end{array}$ & 2 & 2 & 2 & 2 & 1 & 2 & 1 \\
\hline $\begin{array}{l}\text { Galliano-Castillo et al., } \\
2016^{13}\end{array}$ & 2 & 2 & 2 & 2 & 2 & 2 & 2 \\
\hline Gehring et al., $2018^{34}$ & 2 & 2 & 1 & 0 & 2 & 0 & 2 \\
\hline Haggerty et al., $2017^{35}$ & 2 & 2 & 2 & 2 & 2 & 0 & 2 \\
\hline Haggerty et al., $2016^{36}$ & 2 & 2 & 1 & 2 & 2 & 0 & 2 \\
\hline Ji et al., $2019^{37}$ & 2 & 2 & 1 & 2 & 0 & 0 & 2 \\
\hline Kanera et al., $2017^{14}$ & 2 & 2 & 2 & 2 & 0 & 0 & 2 \\
\hline Kanera et al., $2016^{15}$ & 2 & 2 & 2 & 2 & 2 & 0 & 2 \\
\hline Mohamad et al., $2019^{38}$ & 2 & 2 & 2 & 2 & 0 & 0 & 0 \\
\hline Nemli et al., $2019^{26}$ & 2 & 2 & 1 & 2 & 1 & 0 & 2 \\
\hline Short et al., $2017^{23}$ & 2 & 2 & 2 & 2 & 0 & 0 & 0 \\
\hline Uhm et al., $2017^{27}$ & 2 & 2 & 2 & 2 & 2 & 0 & 1 \\
\hline Vallerand et al., $2018^{40}$ & 2 & 2 & 1 & 2 & 2 & 1 & 2 \\
\hline Villaron et al., $2018^{28}$ & 2 & 2 & 1 & 2 & 0 & 0 & 2 \\
\hline \multicolumn{8}{|c|}{$\begin{array}{l}\text { dItem number check list key } \\
1 \text { - Are there clear research questions? } \\
2 \text { - Does the collected data answer the research questions? } \\
3 \text { - Is randomisation appropriately performed? } \\
4 \text { - Are the groups comparable to baseline? } \\
5 \text { - Are there complete outcome data? } \\
6 \text { - Are outcome assessors blinded to the intervention provided? } \\
7 \text { - Did the participants adhere to the assigned intervention? }\end{array}$} \\
\hline
\end{tabular}


A score of 0 indicates a high risk of bias (red), a score of 1 indicates an unclear risk of bias (yellow) and a score of 2 indicates a low risk of bias (green). 
Table 3. Overview of the Key Findings

\begin{tabular}{|c|c|c|c|c|c|}
\hline $\begin{array}{l}\text { Author and } \\
\text { Year }\end{array}$ & Intervention & Physical Outcomes & $\begin{array}{l}\text { Psychosocial } \\
\text { Outcomes }\end{array}$ & $\begin{array}{l}\text { Participant/Clinician Feedback, Preferences } \\
\text { and Technical Issues }\end{array}$ & Key findings \\
\hline $\begin{array}{l}\text { Badger et al., } \\
2017^{16 * *}\end{array}$ & $\begin{array}{l}\text { Six-week programs delivered } \\
\text { over telephone (one } \\
\text { counselling one exercise). }\end{array}$ & NR & $\begin{array}{l}\text { Decrease in } \\
\text { anxiety }(p= \\
<0.002)\end{array}$ & $\mathrm{NR}$ & $\begin{array}{l}\text { Regular social interaction deemed important, telephone } \\
\text { calls linked to adherence to self-managed exercise } \\
\text { program, inclusion of partners in program. Benefits: low } \\
\text { cost, easily implemented. }\end{array}$ \\
\hline $\begin{array}{l}\text { Bantum et al., } \\
2014^{24}\end{array}$ & $\begin{array}{l}\text { Web-based portal to deliver } \\
\text { exercise intervention. } \\
\text { Additional web-platform where } \\
\text { survivors could interact with } \\
\text { one another. }\end{array}$ & $\begin{array}{l}\text { Increased strenuous exercise } \\
\text { min/week }(p=0.01) \\
\text { Increased stretching } \mathrm{min} / \text { week } \\
(p=0.01)\end{array}$ & $\begin{array}{l}\text { Reduced } \\
\text { insomnia }(p= \\
0.03)\end{array}$ & NR & $\begin{array}{l}\text { Web-platform feasible and usable, computer literacy was } \\
\text { considered as important. }\end{array}$ \\
\hline $\begin{array}{l}\text { Bruns et al., } \\
2019^{29}\end{array}$ & $\begin{array}{l}\text { Computer supported strength } \\
\text { training workout. }\end{array}$ & $\begin{array}{l}\text { 4-metre gait speed }+6 \% \text {. } \\
\text { SPPB }+25 \% .\end{array}$ & $\begin{array}{l}\text { Overall quality } \\
\text { of life }+17 \% \text {. }\end{array}$ & $\begin{array}{l}\text { Preference for home program: } 100 \% . \\
\text { Program feedback: overall intervention } \\
\text { grade: } 8 / 10 \text {; clear user interface: } 86 \% \text {. } \\
\text { Difficult to use touch screen on device: } 14 \% .\end{array}$ & $\begin{array}{l}\text { Feasible but requires personalised prehab programs } \\
\text { particularly of older people with complex comorbidities. }\end{array}$ \\
\hline $\begin{array}{l}\text { Cheong et al., } \\
2018^{30}\end{array}$ & $\begin{array}{l}\text { 12-week mobile health } \\
\text { management app + wearable } \\
\text { device with individualised } \\
\text { programs. }\end{array}$ & $\begin{array}{l}\text { Decreased 2MWT time }(p= \\
<0.01) . \\
\text { Decreased } 30 \text { sec STS }(p= \\
<0.01) . \\
\text { Reduced fatigue }(p=0.007) .\end{array}$ & $\begin{array}{l}\text { No statistically } \\
\text { significant } \\
\text { outcomes. }\end{array}$ & NR & $\begin{array}{l}\text { Good compliance, symptom relief, positive results/high } \\
\text { compliance. }\end{array}$ \\
\hline $\begin{array}{l}\text { Cheville et al., } \\
2018^{31}\end{array}$ & $\begin{array}{l}\text { COPE trial, delivered by } \\
\text { physiotherapist and } \\
\text { pedometers. }\end{array}$ & $\begin{array}{l}\text { No statistically significant } \\
\text { outcomes. }\end{array}$ & NR & $\begin{array}{l}\text { Participants that required minimal direction } \\
\text { on how to use module: } 83 \% \text {. }\end{array}$ & $\begin{array}{l}80 \% \text { of participants chose to report via website. No } \\
\text { difficulty in inputting information. Those with less } \\
\text { eHealth literacy required additional assistance initially. }\end{array}$ \\
\hline $\begin{array}{l}\text { Chung et al., } \\
2019^{32}\end{array}$ & $\begin{array}{l}\text { Smartphone app and wearable } \\
\text { smart band. }\end{array}$ & $\begin{array}{l}\text { No statistically significant } \\
\text { outcomes. }\end{array}$ & NR & $\begin{array}{l}\text { Participants that required minimal direction } \\
\text { on how to use module: } 83 \% \text {. }\end{array}$ & $\begin{array}{l}\text { Results indicate the app and phone are both feasible } \\
\text { tools to collect data. }\end{array}$ \\
\hline $\begin{array}{l}\text { Chung et al., } \\
2020^{17}\end{array}$ & Mobile app Walk On & $\begin{array}{l}\text { Increase in total weekly step } \\
\text { count }(p=<0.002)\end{array}$ & NR & NR & $\begin{array}{l}\text { Health promotion using mobile devices can have positive } \\
\text { impact on mental health and physical activity levels. }\end{array}$ \\
\hline $\begin{array}{l}\text { Cnossen et al., } \\
2014^{33}\end{array}$ & $\begin{array}{l}\text { Self-guided program Head } \\
\text { Matters. Phone or email } 10 \\
\text { minutes coaching sessions }\end{array}$ & NR & NR & $\begin{array}{l}\text { Primary barriers reported by participants: } \\
\text { decreased physical condition, treatment } \\
\text { related barriers, emotional problems, lack of } \\
\text { motivation, social barriers and technical } \\
\text { problems. } \\
\text { Primary facilitators reported by participants: } \\
\text { increased physical condition, psychological } \\
\text { wellbeing, feeling motivated, social and } \\
\text { technical facilitators. }\end{array}$ & $\begin{array}{l}\text { Social facilitators: being able to perform exercises at } \\
\text { home or with family, technical facilitators: online or DVD } \\
\text { demonstrations, telephone follow ups, reminders. }\end{array}$ \\
\hline $\begin{array}{l}\text { Eakin et al., } \\
2012^{18}\end{array}$ & $\begin{array}{l}\text { Exercise for Health-rural: trial } \\
\text { evaluating } \\
\text { feasibility/effectiveness of } \\
\text { telephone ex intervention. } \\
\text { Delivered by accredited } \\
\text { exercise physiologist }\end{array}$ & $\begin{array}{l}\text { Increase in strength training 6- } \\
\text { and } 12 \text {-months post } \\
\text { intervention }(p=<0.05) \text {. } \\
\text { Decreased fatigue }(p=<0.001) \text {. } \\
\text { Increased upper body function } \\
(p=<0.001) \text {. }\end{array}$ & $\begin{array}{l}\text { Increased } \\
\text { quality of life } \\
(p=<0.001)\end{array}$ & $\begin{array}{l}\text { Program rated "helpful" to "very helpful" by } \\
89 \% \text { of participants. }\end{array}$ & $\begin{array}{l}\text { Feasible to deliver via telephone to women in remote } \\
\text { regional areas. High participation and very high } \\
\text { retention rates, no serious adverse events, majority of } \\
\text { telephone group participants completing scheduled calls }\end{array}$ \\
\hline
\end{tabular}




\begin{tabular}{|c|c|c|c|c|c|}
\hline $\begin{array}{l}\text { Frensham et al., } \\
2018^{19}\end{array}$ & $\begin{array}{l}\text { Online tool STRIDE (pedometer } \\
+ \text { interactive online resource). } \\
\text { Emailed daily step goals. } \\
\text { Online forum: share } \\
\text { experiences and offer peer } \\
\text { support }\end{array}$ & $\begin{array}{l}\text { Decreased bodily pain }(p= \\
<0.05) \text {. } \\
\text { Increased general health }(p= \\
<0.05) \text {. } \\
\text { Increased physical fitness }(p= \\
<0.01) \text {. } \\
\text { Decreased BP }(p=<0.01) . \\
\text { Decreased waist girth }(p= \\
<0.01) .\end{array}$ & $\begin{array}{l}\text { Increased } \\
\text { social } \\
\text { functioning ( } p \\
=<0.01) . \\
\text { Improved } \\
\text { mental health } \\
(p=<0.05) .\end{array}$ & NR & $\begin{array}{l}\text { No differences between participants in metro vs regional } \\
\text { areas, potential to narrow gap in health status. }\end{array}$ \\
\hline $\begin{array}{l}\text { Galiano-Castillo } \\
\text { et al., } 2017^{12}\end{array}$ & $\begin{array}{l}\text { Internet based exercise } \\
\text { program } 8 \text { weeks (e-CUIDATE). }\end{array}$ & $\begin{array}{l}\text { Improved global health status } \\
(p=<0.001) . \\
\text { Improved physical functioning } \\
(p=<0.001) \text {. } \\
\text { Lower pain severity }(p=0.001) \text {. } \\
\text { Lower pain interference }(p= \\
<0.04) \text {. } \\
\text { Decreased total fatigue }(p= \\
<0.001) \text {. } \\
\text { Increased hand grip strength ( } p \\
=0.006) . \\
\text { Increased back and abdominal } \\
\text { strength ( } p=<0.001) . \\
\text { Increased lower body strength } \\
(p=0.001) .\end{array}$ & $\begin{array}{l}\text { No statistically } \\
\text { significant } \\
\text { outcomes. }\end{array}$ & $\begin{array}{l}\text { Global satisfaction with telehealth program: } \\
98 \% .\end{array}$ & $\begin{array}{l}\text { No health problems or technical issues recorded, } \\
\text { improvements in physical outcomes. }\end{array}$ \\
\hline $\begin{array}{l}\text { Galiano-Castillo } \\
\text { et al., } 2016^{13}\end{array}$ & $\begin{array}{l}\text { Internet based exercise } \\
\text { program } 8 \text { weeks (e-CUIDATE). }\end{array}$ & $\begin{array}{l}\text { Increased 6MWT distance }(p= \\
<0.001) . \\
\text { Improved } \% \text { of predicted } 6 \text { MWT } \\
\text { distance }(p=<0.001) .\end{array}$ & $\begin{array}{l}\text { No statistically } \\
\text { significant } \\
\text { outcomes. }\end{array}$ & $\begin{array}{l}\text { Global satisfaction with telehealth program: } \\
98 \% .\end{array}$ & $98 \%$ for global satisfaction of the intervention. \\
\hline $\begin{array}{l}\text { Garrett et al., } \\
2013^{20}\end{array}$ & $\begin{array}{l}\text { C-STEPS program via telephone } \\
\text { Delivered by psychosocial } \\
\text { oncology counsellors over } 3 \\
\text { months. }\end{array}$ & $\begin{array}{l}\text { Increased physical activity } \\
\text { levels }(p=<0.006)\end{array}$ & $\begin{array}{l}\text { Decrease in } \\
\text { cancer specific } \\
\text { distress }(p= \\
<0.001) . \\
\text { Decreased } \\
\text { stress }(p= \\
<0.001) .\end{array}$ & Mean program satisfaction: 9/10. & $\begin{array}{l}\text { High self-reported satisfaction levels, feasible program } \\
\text { that transcends geographic barriers. }\end{array}$ \\
\hline $\begin{array}{l}\text { Gehring et al., } \\
2018^{34}\end{array}$ & $\begin{array}{l}\mathrm{P} / \mathrm{T} \text { visit at home, } \\
\text { familiarisation with HR monitor } \\
\text { and how to upload data. Data } \\
\text { was monitored by } \mathrm{P} / \mathrm{T} \\
\text { remotely and feedback was } \\
\text { provided via email. More } \\
\text { frequent email or phone } \\
\text { contact was allowed. }\end{array}$ & $\begin{array}{l}\text { Increase in self-reported } \\
\text { physical activity levels from } \\
\text { baseline }(126 \%) . \\
\text { Absolute VO2peak }(p=0.04) \\
\text { Relative VO2peak }(p=0.02) \\
\text { BMI }(p=0.02) \\
\text { Body weight }(p=0.02)\end{array}$ & $\begin{array}{l}\text { No statistically } \\
\text { significant } \\
\text { outcomes. }\end{array}$ & $\begin{array}{l}\text { Participants who rated exercise program as } \\
\text { good or excellent: } 84 \% \text {. }\end{array}$ & $\begin{array}{l}\text { Feasibility of a remotely supervised home-based aerobic } \\
\text { intervention was suitable for motivated patients. No } \\
\text { adverse effects. Participants adhered to } 79 \% \text { of } \\
\text { scheduled sessions. }\end{array}$ \\
\hline $\begin{array}{l}\text { Haggerty et al., } \\
2017^{35}\end{array}$ & $\begin{array}{l}\text { Telemedicine with Wi-Fi scales, } \\
\text { texting. } 15-20 \text { minute }\end{array}$ & $\begin{array}{l}\text { Weight loss }(p=0.02) \\
\text { Decrease in IL2 }(p=<0.05) .\end{array}$ & NR & NR & Feasible, resulted in weight loss/improved QoL. \\
\hline
\end{tabular}




\begin{tabular}{|c|c|c|c|c|c|}
\hline & $\begin{array}{l}\text { telephone counselling sessions } \\
\text { delivered by doctoral students } \\
\text { in clinical psychology and } \\
\text { medical students. }\end{array}$ & & & & \\
\hline $\begin{array}{l}\text { Haggerty et al., } \\
2016^{36}\end{array}$ & $\begin{array}{l}\text { Weekly phone calls, Wi-Fi } \\
\text { tracking and text messages. }\end{array}$ & $\begin{array}{l}\text { Weight loss }(p=0.02) \\
\text { Decrease in IL2 }(p=<0.05)\end{array}$ & NR & NR & $\begin{array}{l}\text { Participants reported interest in programs and felt they } \\
\text { did not require face-to-face visits and were more easily } \\
\text { integrated into daily lives. }\end{array}$ \\
\hline Ji et al., $2019^{37}$ & mHealth program efil breath & $\begin{array}{l}\text { Increased 6MWT distance }(\mathrm{p}= \\
0.001) \text {. }\end{array}$ & $\begin{array}{l}\text { Improved } \\
\text { quality of life } \\
(p=0.002)\end{array}$ & NR & $\begin{array}{l}\text { Only } 40.5 \% \text { of screened patients enrolled. Technology } \\
\text { limitations may have limitations among elderly. }\end{array}$ \\
\hline $\begin{array}{l}\text { Kanera et al., } \\
2017^{14}\end{array}$ & $\begin{array}{l}\text { Cancer Aftercare Guide (web- } \\
\text { based intervention with } 8 \\
\text { modules) }\end{array}$ & $\begin{array}{l}\text { Increase in moderate physical } \\
\text { activity levels }(p=<0.05)\end{array}$ & NR & NR & $\begin{array}{l}\text { Forum for social support and interaction with other } \\
\text { cancer survivors. }\end{array}$ \\
\hline $\begin{array}{l}\text { Kanera et al., } \\
2016^{15}\end{array}$ & $\begin{array}{l}\text { Cancer Aftercare Guide (web- } \\
\text { based intervention with } 8 \\
\text { modules) }\end{array}$ & $\begin{array}{l}\text { Increase in moderate physical } \\
\text { activity levels }(p=<0.05)\end{array}$ & NR & NR & $\begin{array}{l}\text { Intervention was effective at increasing and maintaining } \\
\text { moderate PA amongst early cancer survivors. }\end{array}$ \\
\hline $\begin{array}{l}\text { Ligibel et al., } \\
2010^{25}\end{array}$ & $\begin{array}{l}\text { 12-week telephone exercise } \\
\text { intervention during treatment } \\
\text { by AEP. Pedometer and } \mathrm{HR} \\
\text { monitor. }\end{array}$ & $\begin{array}{l}\text { Increased weekly physical } \\
\text { activity levels }(p=<0.001) \\
\text { Improved cardiorespiratory } \\
\text { fitness }(p=<0.003)\end{array}$ & $\begin{array}{l}\text { Improved } \\
\text { quality of life } \\
(p=0.001)\end{array}$ & NR & $\begin{array}{l}\text { Improved weekly exercise and improved QoL, fatigue, } \\
\text { fitness. }\end{array}$ \\
\hline $\begin{array}{l}\text { Mohamad et } \\
\text { al., } 2019^{38}\end{array}$ & $\begin{array}{l}\text { Pedometer and access to } \\
\text { online diet/PA resources. }\end{array}$ & Decrease in weight $(p=<0.001)$ & $\begin{array}{l}\text { No statistically } \\
\text { significant } \\
\text { outcomes. }\end{array}$ & NR & Weight loss linked to intervention group at 12 months. \\
\hline $\begin{array}{l}\text { Nemli et al., } \\
2019^{26}\end{array}$ & Telephone calls with $\mathrm{P} / \mathrm{T}$. & $\begin{array}{l}\text { Increased weekly physical } \\
\text { activity levels }(p=0.001)\end{array}$ & $\begin{array}{l}\text { Increased } \\
\text { quality of life } \\
(p=<0.05)\end{array}$ & NR & Feasible with positive physical and QOL outcomes. \\
\hline $\begin{array}{l}\text { Quintiliani et } \\
\text { al., } 2016^{21}\end{array}$ & $\begin{array}{l}\text { mHealth (text messaging, } \\
\text { wireless devices to track } \\
\text { weight/step and telephone } \\
\text { sessions). }\end{array}$ & $\begin{array}{l}\text { No statistically significant } \\
\text { outcomes. }\end{array}$ & $\begin{array}{l}\text { No statistically } \\
\text { significant } \\
\text { outcomes. }\end{array}$ & $\begin{array}{l}\text { Likely to participate again: } 90 \% \text {. } \\
\text { Rated calls helpful: } 100 \% \text {. } \\
\text { Somewhat or not at all likely to participate } \\
\text { again if the program cost: } 70 \% \text {. }\end{array}$ & $\begin{array}{l}\text { Select devices that don't rely on WiFi and look at offering } \\
\text { low cost smartphones, larger studies. Human based } \\
\text { interactions were considered important. }\end{array}$ \\
\hline $\begin{array}{l}\text { Rocque et al., } \\
2017^{22}\end{array}$ & $\begin{array}{l}\text { Pedometer and access to } \\
\text { online diet/PA resources. }\end{array}$ & $\begin{array}{l}\text { No statistically significant } \\
\text { outcomes }\end{array}$ & $\begin{array}{l}\text { No statistically } \\
\text { significant } \\
\text { outcomes. }\end{array}$ & $\begin{array}{l}\text { Likely to participate again: } 90 \% \text {. } \\
\text { Rated calls helpful: } 100 \% \text {. } \\
\text { Somewhat or not at all likely to participate } \\
\text { again if the program cost: } 70 \% \text {. }\end{array}$ & $\begin{array}{l}\text { Only } 34 \% \text { of participants chose to participate. }>50 \% \text { of } \\
\text { those who enrolled finished }\end{array}$ \\
\hline $\begin{array}{l}\text { Short et al., } \\
2017^{23}\end{array}$ & $\begin{array}{l}\text { Online modules, email } \\
\text { reminders. }\end{array}$ & $\begin{array}{l}\text { Increase in moderate-vigorous } \\
\text { aerobic physical activity. }\end{array}$ & $\begin{array}{l}\text { No statistically } \\
\text { significant } \\
\text { outcomes. }\end{array}$ & $\begin{array}{l}\text { Usability of website: adequate. } \\
\text { Website acceptability score: } 22 / 36 \text {. }\end{array}$ & $\begin{array}{l}\text { Highlights the need for cost effective, low burden } \\
\text { interventions }\end{array}$ \\
\hline $\begin{array}{l}\text { Timmerman et } \\
\text { al., } 2017^{39}\end{array}$ & $\begin{array}{l}\text { RMT application: modules with } \\
\text { remote supervision by } \mathrm{P} / \mathrm{T} \text {. }\end{array}$ & NR & NR & $\begin{array}{l}\text { Mean module satisfaction: } 5 / 7 \text {. } \\
\text { Qualitative data indicated lack of } \\
\text { satisfaction with feedback on progress from } \\
\text { physician which decreased motivation. } \\
\text { Points to improve usability: connection } \\
\text { issues between equipment and portal and }\end{array}$ & $\begin{array}{l}\text { Active involvement of HCP is considered essential for } \\
\text { successful implementation of telehealth care }\end{array}$ \\
\hline
\end{tabular}




\begin{tabular}{|c|c|c|c|c|c|}
\hline & & & & $\begin{array}{l}\text { improving visualisation of results in the } \\
\text { online portal. } \\
\text { Physiotherapists voiced a positive intention } \\
\text { to keep using the web-based modules. } \\
\text { Other health professional comments: web- } \\
\text { based module may contribute to improved } \\
\text { accessibility of a cancer rehabilitation } \\
\text { program. } \\
\text { Smartphone application considered to } \\
\text { improve program accessibility even more. } \\
\text { Suggestions for future programs: a blended } \\
\text { care approach of supervised and home- } \\
\text { based training. }\end{array}$ & \\
\hline $\begin{array}{l}\text { Uhm et al., } \\
2017^{27}\end{array}$ & $\begin{array}{l}\text { mHealth app (Smart After } \\
\text { Care) with pedometer. }\end{array}$ & $\begin{array}{l}\text { Improved function in physical, } \\
\text { role, emotional, cognitive, } \\
\text { sexual functioning and future } \\
\text { perspective scales ( } p=<0.05 \text { ). }\end{array}$ & $\begin{array}{l}\text { Improved } \\
\text { function in } \\
\text { physical, role, } \\
\text { emotional, } \\
\text { cognitive, } \\
\text { sexual } \\
\text { functioning } \\
\text { and future } \\
\text { perspective } \\
\text { scales ( } p= \\
<0.05) \text {. } \\
\end{array}$ & NR & $\begin{array}{l}\text { Personalised programs needed. Clear exclusion of } \\
\text { participants due to lack of access, consider how to } \\
\text { overcome this barrier in future studies. }\end{array}$ \\
\hline $\begin{array}{l}\text { Vallerand et al., } \\
2019^{40}\end{array}$ & $\begin{array}{l}\text { 12-week exercise telephone } \\
\text { counselling intervention. }\end{array}$ & $\begin{array}{l}\text { Increased aerobic exercise } \\
\text { participation }(p=<0.001) \text {. }\end{array}$ & $\begin{array}{l}\text { Increased } \\
\text { instrumental } \\
\text { and affective } \\
\text { attitude ( } \mathrm{p}= \\
0.02 \text { ). } \\
\text { Increased } \\
\text { coping } \\
\text { planning ( } \mathrm{p}= \\
<0.001) .\end{array}$ & NR & $\begin{array}{l}\text { No adverse events, adherence to telephone calls was } \\
93 \% \text {. Doubled PA compared to self-directed group. }\end{array}$ \\
\hline $\begin{array}{l}\text { Villaron et al., } \\
2018^{28}\end{array}$ & $\begin{array}{l}\text { Weekly SMS text for exercise } \\
\text { promotion, pedometer. }\end{array}$ & $\begin{array}{l}\text { Improved self-reported fatigue } \\
(p=0.01) \\
\text { Improved physical capacity }(p= \\
<0.01)\end{array}$ & $\begin{array}{l}\text { Improved } \\
\text { quality of life } \\
(p=0.01)\end{array}$ & NR & $\begin{array}{l}\text { Feasibility and adherence was good, credible alternative } \\
\text { to supervised programs }\end{array}$ \\
\hline
\end{tabular}

*Statically significant $(\mathrm{p}=<0.05)$ **Partners not included in data extraction.

NR: Not Reported; SPPB: Short Performance Physical Battery; 2MWT: Two Minute Walk Test; STS: Sit-to-Stand; COPE: Collaborative Care to Preserve Performance in Cancer; STRIDE: Steps Towards Improving Diet and Exercise; BP: Blood Pressure; e-CUIDATE: Telehealth System; 6MWT: Six Minute Walk Test; C-STEPS: Cancer Survivor Telephone Education and Personal Support; N/A: Not applicable; P/T: Physiotherapist; HR: Heart Rate; VO2: Oxygen Consumption; BMI: Body Max Index; IL2: Inter-leukin-2; mHealth: Mobile Health; PA: Physical Activity; RMT: Remote Monitoring and Treatment; HCP: Health Care Professional; SMS: Short Message Service 
Web-based platform and the impact on physical and psychosocial outcomes

Evidence identified a range of physical and psycho-social benefits of delivering an online web-based exercise intervention for people affected by cancer. Physical benefits included increased ability and performance across a range of activities including: strenuous exercise, ${ }^{24}$ muscle stretching, ${ }^{24} 4$-metre gait speed, ${ }^{29}$ grip strength, ${ }^{12}$ six-minute walk test. ${ }^{12,37}$ Participants reported decreased bodily pain, ${ }^{12,29}$ increased general health ${ }^{12,29}$ and physical functioning, ${ }^{12,29}$ reduced blood pressure (BP), ${ }^{19}$ waist girth ${ }^{19}$ and fatigue. ${ }^{12}$ Other studies observed weight loss ${ }^{35,38}$ and decreased interleukin levels ${ }^{35}$ with improvements in overall PA levels. ${ }^{14,15,23}$ However, several studies did not observe any statistically significant improvements in physical outcomes. ${ }^{31,22,39}$

Web-based exercise interventions demonstrated improvements in psycho-social outcomes, including: insomnia, ${ }^{24}$ overall quality of life,${ }^{29,37}$ social functioning ${ }^{19}$ and mental well-being. ${ }^{19}$ Noteworthy, the majority of the web-based intervention studies did not observe improvements in psycho-social outcomes. ${ }^{12,14,15,22,23,31,35,38,39}$

\section{Mobile Apps and the impact on physical and psychosocial outcomes}

Across the 29 included studies, four studies explored the impact of exercise apps on physical and psychological outcomes. Improvements were observed in the sit-to-stand test, twominute walk test, ${ }^{30}$ and overall increased total weekly step count. ${ }^{32}$ Only one study reported improved psycho-social outcomes across the following domains, emotional, cognitive, sexual functioning. ${ }^{28}$ 
Ten studies used telephone counselling communication. ${ }^{16,18,20,21,25,26,33-35,40}$ The studies reported inconsistent findings in relation to the relationship of the exercise telephone counselling interventions across a range of physical outcomes. Several studies improvements in fatigue levels, upper body function, ${ }^{18}$ increased PA levels, ${ }^{14,20,25,26,34,40,}$ improved cardiovascular fitness, ${ }^{25}$ reduced body weight ${ }^{20,35}$ and reduced interleukin levels. ${ }^{35}$ However, three studies did not observe any changes in physical outcomes. ${ }^{16,21,33}$ Similar with the physical outcomes, the relationships between the telephone exercise interventions and psycho-social outcomes were varied. Four studies identified no statistically significant relationship with psycho-social outcomes. ${ }^{21,33-35}$ However, other studies reported reduced anxiety and depression, ${ }^{16}$ improved quality of life,${ }^{18,25,26}$ reduced stress,${ }^{20}$ and improved coping strategies ${ }^{40}$ in favour of the telephone exercise intervention.

\section{SMS messaging and the impact on physical and psychosocial outcomes}

Only one study used weekly SMS message communication which demonstrated improved fatigue levels and physical capacity, with overall improvements in quality of life. ${ }^{28}$

\section{Feasibility of telehealth exercise interventions in cancer care}

The feasibility of the different modalities of exercise telehealth interventions varied, see

Table 4. Feasibility was determined by the percentage of participants who completed and adhered to the prescribed intervention, in addition to eligible participants who declined to participate. The completion rates of the entire web-based exercise inventions ranged from 11$100 \%$, with 24 out of the 29 studies reporting completion rates over $70 \%$. The studies with completion rates less than $70 \%$ were telephone, mobile app and web-based 
interventions. $^{22,23,32,33,37}$ Adherence to the allocated interventions ranged from $53-100 \%$, with many studies not reporting total adherence rates. The studies with the highest adherence rates to the allocated intervention were telephone and web based interventions. ${ }^{16,32,29,34}$ The most frequent reasons for non-participation were: did not meet inclusion criteria, did not enrol, declined to participate and the participant could not be contacted. Lack of access to specific devices such as smartphones or computers and poor computer literacy were also reported as reasons eligible participants did not enrol in a study. ${ }^{14,20,21-23}$ Health and medical concerns or cancer-related barriers only prevented a small number of eligible participants from participating. ${ }^{12,13,18,39}$ 
Table 4. Feasibility of Exercise Interventions

\begin{tabular}{|c|c|c|c|c|c|}
\hline Author and Year & $\begin{array}{l}\text { Completion of entire } \\
\text { intervention }(\% \text { of } \\
\text { sample) }\end{array}$ & $\begin{array}{l}\text { Adherence to intervention (\% of } \\
\text { sample) }\end{array}$ & $\begin{array}{l}\text { Eligible participants who did not } \\
\text { participate (n) } \\
\text { Reasons (if provided) }\end{array}$ & $\begin{array}{l}\text { Average completion } \\
\text { and adherence rates }\end{array}$ & Mode and intensity of exercise \\
\hline $\begin{array}{l}\text { Badger et al., } \\
2017^{16}\end{array}$ & $100 \%$ & Adherence to all sessions: $100 \%$ & $\begin{array}{l}\text { Total eligible participants } \mathrm{n}=165 \\
\text { Did not enrol } \mathrm{n}=68 \\
\text { Reasons: } \\
\text { Did not meet inclusion criteria } \mathrm{n}= \\
25 \\
\text { Declined to participate } \mathrm{n}=18 \\
\text { Did not speak English } \mathrm{n}=25\end{array}$ & $100 \%$ & Low impact exercise. \\
\hline $\begin{array}{l}\text { Bantum et al., } \\
2014^{24}\end{array}$ & $89 \%$ & Adherence to all sessions: $67 \%$. & $\begin{array}{l}\text { Total eligible participants } \mathrm{n}=623 \\
\text { Did not enrol } \mathrm{n}=271 \\
\text { Reasons: } \\
\text { Did not meet inclusion criteria } \mathrm{n}= \\
74 \\
\text { Dropped out or refused } \mathrm{n}=11 \\
\text { Left name but did not respond } \\
\text { further } \mathrm{n}=186\end{array}$ & $78 \%$ & NR \\
\hline Bruns et al., $2019^{29}$ & $100 \%$ & Adherence to all exercises: $86 \%$. & $\begin{array}{l}\text { Total eligible participants } \mathrm{n}=24 \\
\text { Did not enrol } \mathrm{n}=10 \\
\text { Reasons: } \\
\text { Personal reasons } \mathrm{n}=2 \\
\text { Already scheduled for surgery }<14 \\
\text { days } \mathrm{n}=2 \\
\text { Preferred direct surgery } \mathrm{n}=6 \\
\end{array}$ & $93 \%$ & Bodyweight strength training. \\
\hline $\begin{array}{l}\text { Cheong et al., } \\
2018^{30}\end{array}$ & $74 \%$ & NR & $\begin{array}{l}\text { Total eligible participants: } n=102 \\
\text { Did not enrol } n=0\end{array}$ & $74 \%$ & $\begin{array}{l}\text { Aerobic, strength, flexibility and } \\
\text { pelvic floor muscle training. }\end{array}$ \\
\hline $\begin{array}{l}\text { Cheville et al., } \\
2018^{31}\end{array}$ & $85 \%$ & NR & $\begin{array}{l}\text { Total eligible participants } n=516 \\
\text { Did not enrol } n=0\end{array}$ & $85 \%$ & $\begin{array}{l}\text { Strength, aerobic, flexibility and } \\
\text { balance training. }\end{array}$ \\
\hline $\begin{array}{l}\text { Chung et al., } \\
2019^{32}\end{array}$ & $79 \%$ & $\begin{array}{l}\text { Compliance for smartphone app: } \\
88 \% \text {. } \\
\text { Compliance for smart bands: } 53 \% \text {. }\end{array}$ & $\begin{array}{l}\text { Total eligible participants } \mathrm{n}=656 \\
\text { Did not enrol } \mathrm{n}=496 \\
\text { Reasons: } \\
\text { Declined to participate } \mathrm{n}=320 \\
\text { Could not contact } \mathrm{n}=176\end{array}$ & $73 \%$ & Walking (aerobic) training. \\
\hline $\begin{array}{l}\text { Chung et al., } \\
2020^{17}\end{array}$ & $58 \%$ & NR & $\begin{array}{l}\text { Total eligible participants } \mathrm{n}=212 \\
\text { Did not enrol } \mathrm{n}=148 \\
\text { Reasons: }\end{array}$ & $58 \%$ & Walking (aerobic) training. \\
\hline
\end{tabular}




\begin{tabular}{|c|c|c|c|c|c|}
\hline & & & $\begin{array}{l}\text { Declined to participate } n= \\
108 \text { Could not contact } n=40\end{array}$ & & \\
\hline $\begin{array}{l}\text { Cnossen et al., } \\
2014^{33}\end{array}$ & $64 \%$ & NR & $\begin{array}{l}\text { Total eligible participants } \mathrm{n}=41 \\
\text { Did not enrol } \mathrm{n}=7 \\
\text { Reasons } \\
\text { Declined to participate } \mathrm{n}=34\end{array}$ & $64 \%$ & Mobility training. \\
\hline Eakin et al., $2012^{18}$ & $96 \%$ & NR & $\begin{array}{l}\text { Total eligible participants } \mathrm{n}=234 \\
\text { Did not enrol } \mathrm{n}=79 \\
\text { Reasons: } \\
\text { Health and medical concerns } \mathrm{n}=36 \\
\text { Difficult to contact } \mathrm{n}=8 \\
\text { Not interested } \mathrm{n}=15 \\
\text { No reason given } \mathrm{n}=12 \\
\text { Other } \mathrm{n}=8\end{array}$ & $96 \%$ & Aerobic and strength training. \\
\hline $\begin{array}{l}\text { Frensham et al., } \\
2018^{19}\end{array}$ & $76 \%$ & NR & $\begin{array}{l}\text { Total eligible participants } \mathrm{n}=169 \\
\text { Did not enrol } \mathrm{n}=74 \\
\text { Reasons } \\
\text { Declined to participate } \mathrm{n}=17 \\
\text { Did not meet inclusion criteria } \mathrm{n}= \\
57\end{array}$ & $76 \%$ & Walking (aerobic) training. \\
\hline $\begin{array}{l}\text { Galiano-Castillo et } \\
\text { al., } 2017^{12}\end{array}$ & $94 \%$ & NR & $\begin{array}{l}\text { Total eligible participants } \mathrm{n}=99 \\
\text { Did not enrol } \mathrm{n}=18 \\
\text { Reasons } \\
\text { Cancer recurrence } \mathrm{n}=3 \\
\text { No internet access } \mathrm{n}=2 \\
\text { Personal reasons }=5 \\
\text { Distance } \mathrm{n}=5 \\
\text { Other reasons } \mathrm{n}=3\end{array}$ & $94 \%$ & Aerobic and resistance training. \\
\hline $\begin{array}{l}\text { Galiano-Castillo et } \\
\text { al., } 2016^{13}\end{array}$ & $94 \%$ & NR & $\begin{array}{l}\text { Total eligible participants } \mathrm{n}=99 \\
\text { Did not enrol } \mathrm{n}=18 \\
\text { Reasons } \\
\text { Cancer recurrence } \mathrm{n}=3 \\
\text { No internet access } \mathrm{n}=2 \\
\text { Personal reasons }=5 \\
\text { Distance } \mathrm{n}=5 \\
\text { Other reasons } \mathrm{n}=3\end{array}$ & $94 \%$ & Aerobic and resistance training. \\
\hline $\begin{array}{l}\text { Garrett et al., } \\
2013^{20}\end{array}$ & $70 \%$ & NR & NR & $70 \%$ & NR. \\
\hline $\begin{array}{l}\text { Gehring et al., } \\
2018^{34}\end{array}$ & $83 \%$ & $\begin{array}{l}\text { Completion of sessions: } 70 \% \\
\text { completed } \geq 75 \% \text { of prescribed } \\
\text { exercise sessions. }\end{array}$ & $\begin{array}{l}\text { Total eligible participants } \mathrm{n}=136 \\
\text { Did not enrol } \mathrm{n}=66 \\
\text { Reasons: } \\
\text { No reason reported } \mathrm{n}=18\end{array}$ & $77 \%$ & Moderate intensity aerobic training. \\
\hline
\end{tabular}




\begin{tabular}{|c|c|c|c|c|c|}
\hline & & & $\begin{array}{l}\text { No response } \mathrm{n}=8 \\
\text { Inaccessible } \mathrm{n}=4 \\
\text { No time } \mathrm{n}=18 \\
\text { Lack of motivation } \mathrm{n}=6 \\
\text { Physical limitations } \mathrm{n}=4 \\
\text { Language barriers } \mathrm{n}=2 \\
\text { No cognitive complaints } \mathrm{n}=2 \\
\text { Progressive disease } \mathrm{n}=2 \\
\text { Other } \mathrm{n}=2\end{array}$ & & \\
\hline $\begin{array}{l}\text { Haggerty et al., } \\
2017^{35}\end{array}$ & $78 \%$ & NR & $\begin{array}{l}\text { Total eligible participants } \mathrm{n}=131 \\
\text { Did not enrol } \mathrm{n}=90 \\
\text { Reasons: } \\
\text { Questionnaire incomplete } \mathrm{n}=4 \\
\text { Not interested } \mathrm{n}=64 \\
\text { Enrolment goal for site met } \mathrm{n}=2 \\
\text { Other } \mathrm{n}=20\end{array}$ & $78 \%$ & NR. \\
\hline $\begin{array}{l}\text { Haggerty et al., } \\
2016^{36}\end{array}$ & $90 \%$ & NR & $\begin{array}{l}\text { Total eligible participants } \mathrm{n}=172 \\
\text { Did not enrol } \mathrm{n}=91 \\
\text { Reasons not provided }\end{array}$ & $90 \%$ & NR. \\
\hline Ji et al., $2019^{37}$ & $67 \%$ & NR & NR & $67 \%$ & NR. \\
\hline $\begin{array}{l}\text { Kanera et al., } \\
2017^{14}\end{array}$ & $89 \%$ & NR & $\begin{array}{l}\text { Total eligible participants } \mathrm{n}=1303 \\
\text { Did not enrol } \mathrm{n}=785 \\
\text { Reasons: } \\
\text { Declined to participate } \mathrm{n}=100 \\
\text { Did not meet inclusion criteria } \mathrm{n}=5 \\
\text { Computer literacy } \mathrm{n}=10 \\
\text { Unknown } \mathrm{n}=670\end{array}$ & $89 \%$ & $\begin{array}{l}\text { Moderate to vigorous intensity } \\
\text { aerobic training. }\end{array}$ \\
\hline $\begin{array}{l}\text { Kanera et al., } \\
2016^{15}\end{array}$ & $89 \%$ & NR & $\begin{array}{l}\text { Total eligible participants } \mathrm{n}=1303 \\
\text { Did not enrol } \mathrm{n}=785 \\
\text { Reasons: } \\
\text { Declined to participate } \mathrm{n}=100 \\
\text { Did not meet inclusion criteria } \mathrm{n}=5 \\
\text { Computer literacy } \mathrm{n}=10 \\
\text { Unknown } \mathrm{n}=670\end{array}$ & $89 \%$ & $\begin{array}{l}\text { Moderate to vigorous intensity } \\
\text { aerobic training. }\end{array}$ \\
\hline $\begin{array}{l}\text { Ligibel et al., } \\
2010^{25}\end{array}$ & $83 \%$ & NR & NR & $83 \%$ & Moderate intensity aerobic training. \\
\hline $\begin{array}{l}\text { Mohamad et al., } \\
2019^{38}\end{array}$ & $92 \%$ & Accessed online resources: $58 \%$. & NR & $75 \%$ & Walking (aerobic training) \\
\hline Nemli et al., $2019^{26}$ & $100 \%$ & NR & NR & $100 \%$ & $\begin{array}{l}\text { Moderate intensity aerobic training } \\
\text { and walking. }\end{array}$ \\
\hline $\begin{array}{l}\text { Quintiliani et al., } \\
2016^{21}\end{array}$ & $100 \%$ & NR & $\begin{array}{l}\text { Total eligible participants } n=27 \\
\text { Did not enrol } n=14\end{array}$ & $100 \%$ & NR. \\
\hline
\end{tabular}




\begin{tabular}{|c|c|c|c|c|c|}
\hline & & & $\begin{array}{l}\text { Reasons: } \\
\text { Not having home WiFi and/or } \\
\text { smartphone } n=9 \\
\text { Not being overweight or obese } n= \\
3 \\
\text { Other } n=1\end{array}$ & & \\
\hline $\begin{array}{l}\text { Rocque et al., } \\
2017^{22}\end{array}$ & $45 \%$ & NR & $\begin{array}{l}\text { Total eligible participants } \mathrm{n}=119 \\
\text { Did not enrol } \mathrm{n}=79 \\
\text { Reasons not provided }\end{array}$ & $45 \%$ & NR. \\
\hline Short et al., $2017^{23}$ & $11 \%$ & NR & $\begin{array}{l}\text { Total eligible participants } \mathrm{n}=593 \\
\text { Did not enrol } \mathrm{n}=101 \\
\text { Reasons not provided }\end{array}$ & $11 \%$ & $\begin{array}{l}\text { Moderate intensity aerobic training } \\
\text { and walking. }\end{array}$ \\
\hline $\begin{array}{l}\text { Timmerman et al., } \\
2017^{39}\end{array}$ & $76 \%$ & NR & NR & $76 \%$ & $\begin{array}{l}\text { Individually tailored program, } \\
\text { type/intensity NR. }\end{array}$ \\
\hline Uhm et al., $2017^{27}$ & $95 \%$ & NR & NR & $95 \%$ & $\begin{array}{l}\text { Moderate intensity aerobic and } \\
\text { strength. }\end{array}$ \\
\hline $\begin{array}{l}\text { Vallerand et al., } \\
2019^{40}\end{array}$ & $93 \%$ & NR & NR & $93 \%$ & Aerobic training. \\
\hline $\begin{array}{l}\text { Villaron et al., } \\
2018^{28}\end{array}$ & $72 \%$ & Participation rate: $71 \%$. & NR & $72 \%$ & NR. \\
\hline
\end{tabular}




\section{DISCUSSION}

People diagnosed with cancer require regular appropriately prescribed exercise as part of their treatment and beyond for optimum health related outcomes, reduction in treatment related side effects and sustained or improved quality of life. Socially distant face-to-face services remain best practice but due to the COVID-19 pandemic people who are immunocompromised are being advised or are choosing to remain in the home. Therefore, this timely systematic review has provided clinicians with evidence-based recommendations regarding the use of telehealth as a platform to deliver and receive exercise services for people affected by cancer. Interestingly, none of the included studies in this review reported using face-to-face telehealth platforms such as Zoom, Skype, Microsoft Teams or FaceTime. The reviewers recommend that further research is needed to understand the barriers and facilitators to the delivery of exercise services via online video conferencing using such platforms as well as the experiences of the participants involved in these interventions. It is important to note that some studies combined interventions using multiple telehealth approaches, making it difficult to make conclusions regarding the feasibility or favourability of one modality over another. However, across all of the included studies a range of beneficial outcomes were reported across physical and psycho-social measures following the delivery of exercise interventions via telehealth.

\section{Clinician experiences}

During the current COVID-19 pandemic, many clinicians have had to move from fully faceto-face services to telehealth only services very quickly internationally. ${ }^{41}$ However, this review has underscored that little is known about the barriers and facilitator of telehealth in service delivery, or the experiences from the healthcare professional's perspective. Only one study was identified in this rapid review which explored clinician experiences (specifically from physiotherapists). ${ }^{39}$ This study reported that to improve accessibility a smartphone supported application combined with face-to-face training and home-based training worked best. Overall feedback from therapists showed positive responses, citing that the Telehealth intervention improved accessibility and support for individuals diagnosed with cancer. ${ }^{39}$ Other challenges might include the inability to see 360 degrees around the patient and access to exercise and monitoring equipment within the home setting. More research is required to 
understand the experience of clinicians in delivering exercise via telehealth platforms without the use of face to face services.

\section{Participant experience and barriers to participation}

There has been significant development in the transition to online platforms since the COVID-19 pandemic. This has resulted in many more people of all ages who have adopted these technologies for exercise classes and connecting with people. ${ }^{42}$ However, little is known about what specific challenges maybe experienced by users. It also needs to be considered that some individuals may have poor technology literacy and access due to financial or connectivity issues (these individuals often are a high risk for illness) and may require additional support and training. Common problems identified have included difficulties accessing programs or websites via mobile phone, a lack of individualised exercise programs and the absence of interactions with clinicians. ${ }^{39}$ Technical problems are a common issue and one solution could be to implement a face-to-face familiarisation session, training and to pilot testing web-based modules or smart phone applications prior to rolling out the program across larger cohorts. ${ }^{39}$ Telehealth can relies on web networks with varying speeds and capacities which are difficult problems to provide solutions to in practice. As the demand for online video conferencing platforms continues to increase internationally, so will customer feedback which may provide a means to technical improvements through frequent platform updates.

\section{Recommendations for future telehealth interventions}

Current growth in use of technology to connect and deliver cancer services has become more and more prevalent within our society due to the COVID-19 pandemic. ${ }^{42,43}$ Platforms for video conferencing have already seen exponential growth and improvements have been implemented to support the demand for the services. Future research should be considered to identify the most beneficial, free and accessible video conferencing platforms to deliver optimal exercise telehealth services compliant with data protection. Exercise interventions must carefully consider the need for training and access to optimum internet servers to minimise disruption to the service due to connectivity issues. Clinicians should consider the needs of the participant (equipment and familiarisation) and the information required for them to exercise at home in front of a screen successfully. Social connection can also be facilitated using this video platforms compared to traditional telephone delivery and requires 
further research. Given the global impact due to COVID-19 and the negative impact that this experience has had on individuals diagnosed with cancer, ${ }^{44}$ this rapid review is timely to assist healthcare professionals on understanding the feasibility of exercise telehealth interventions and the physical and psycho-social outcomes of such interventions. Future research is recommended to understand the experiences of healthcare professionals involved with the delivery of Telehealth cancer services. Further insight into the experience of healthcare professionals may identify the barriers and facilitators of telehealth service to optimise future cancer services. Given the vital role healthcare professionals play in the development and administration of Telehealth programs, future studies may also consider a qualitative approach to allow for an in-depth exploration of clinician experience and feedback. By combining both clinician and participant feedback, telehealth programs can be designed to improve adherence rates by removing potential barriers to optimise quality of life, physical and psycho-social outcomes. Further research is also warranted to explore the feasibility of exercise interventions delivered live by allied health and medical professionals via online video conferencing platforms and whether telehealth clinician training is warranted for the successful delivery of exercise services. It is likely given the global climate that more research will be published on the important topic of telehealth in cancer care in the future.

\section{IMPLICATIONS FOR NURSING PRACTICE}

Telehealth is rapidly emerging as a critical tool for cancer care from prevention ${ }^{45}$ to palliation. ${ }^{46}$ Oncology nurses recognise that patients with cancer want to stay well and limit their time in the outpatient and inpatient medical settings, particularly during COVID-19, and telehealth can help patients stay well while staying closer to home and living their lives. Telehealth may afford solutions to optimise the coordination of cancer care with reduced costs, time savings, and increased access to care, and person-centred care in the community setting. To observe these successes in oncology practice, healthcare professionals must focus on appropriate training, education, as well as address important gaps such as those inherent in the digital divide.

\section{CONCLUSION}

This rapid review highlighted that telehealth is a feasible alternative for the delivery of exercise interventions for individuals diagnosed with cancer. Telehealth has its own inherent challenges and will never completely replace face-to-face exercise delivery. This review has 
provided clinicians with the most up-to-date evidence regarding the feasibility and associated physical and psycho-social benefits to exercise telehealth interventions. Exercise telehealth interventions can provide a safe delivery alternative during the 2020 global COVID-19 pandemic and feasible platform moving forward in the future provision of cancer services. The widespread adoption of Telehealth may provide individuals in rural or remote areas or those with severe immunosuppression the opportunity to access supervised exercise therapy which they may not have had otherwise. More research is required to assess the feasibility of telehealth platforms such as Zoom, Skype, Microsoft Teams or FaceTime, to determine the overall participant and exercise professional telehealth exercise delivery experience to assist in the development of training, professional development and telehealth exercise guidelines.

\section{Conflict of interest statement}

The authors declare that there are no conflicts of interest in relation to this work.

\section{Funding source}

This research did not receive any specific grant from funding agencies in the public, commercial or not-for-profit sectors. 


\section{REFERENCES}

1. Hayes SC, Newton RU, Spence RR, Galvão DA. The Exercise and Sports Science Australia position statement: Exercise medicine in cancer management. J Sci Med Sport. 2019;22(11):1175-1199. DOI: https://doi.org/10.1016/j.jsams.2019.05.003

2. Smith AC, Thomas E, Snoswell CL, Haydon H, Mehrotra A, Clemensen J, Caffery LJ. Telehealth for global emergencies: Implications for coronavirus disease 2019 (COVID-19). $J$ Telemed 2020.2 DOI: https://doi.org/10.1177/1357633X20916567

3. Department of Health. Australian Government Department of Health Website [Internet]. Canberra: Department of Health; 2015 [updated 2015 Apr 07; cited 2020 May 12]. Available from: https://www1.health.gov.au/internet/main/publishing.nsf/Content/e-healthtelehealth

4. Cox A, Lucas G, Marcu A, Piano M, Grosvenor W, Mold F, et al. Cancer Survivors' Experience With Telehealth: A Systematic Review and Thematic Synthesis. J Med Internet Res. 2017;19(1):e11. DOI: 10.2196/jmir.6575

5. Peretti A, Amenta F, Tayebati SK, Nittari G, Mahdi SS. Telerehabilitation: review of the state-of-the-art and areas of application. JMIR RATE. 2017;4(2):e7. DOI: 10.2196/rehab.7511

6. Pizzo PA. Management of Patients With Fever and Neutropenia Through the Arc of Time: A Narrative Review. Ann Intern Med. 2019;170(6):389-97. DOI: https://doi.org.10.7326/M18-3192.

7. Hanna TP, Evans GA, Booth CM. Cancer, COVID-19 and the precautionary principle: prioritizing treatment during a global pandemic. Nat Rev Clin Oncol. 2020 May;17(5):268-70. DOI: https://doi.org/10.1038/s41571-020-0362-6

8. Paterson C, Gobel B, Gosselin T, Haylock PJ, Papadopoulou C, Slusser K, Rodriguez A, Pituskin E. Oncology nursing during a pandemic: critical reflections in the context of COVID-19. Sem in Oncol Nurs. 2020 Apr 23; 36(3):151028. DOI: 10.1016/j.soncn.2020.151028

9. World Health Organisation. Coronavirus Disease (COVID-19) Situation Report 189. 2020 Jul 27 [cited 2020 July 28]. Available from: https://www.who.int/docs/default-source/coronaviruse/situationreports/20200727-covid-19-sitrep-189.pdf?sfvrsn=b93a6913_2 
10. Hong QN, Pluye P, Fàbregues S, Bartlett G, Boardman F, Cargo M, Dagenais P, Gagnon M-P, Griffiths F, Nicolau B, O’Cathain A, Rousseau M-C, Vedel I. Mixed Methods Appraisal Tool (MMAT), version 2018. Registration of Copyright (\#1148552), Canadian Intellectual Property Office, Industry Canada.

11. JBI Manual for Evidence Synthesis. The Narrative Synthesis of Data. 2020 [cited 2020 Sept 11]. Available from: https://wiki.jbi.global/display/MANUAL/7.3.6.5.2+The+narrative+synthesis+of+ data

12. Galiano-Castillo N, Arroyo-Morales M, Lozano-Lozano M, Fernández-Lao C, Martín-Martín L, Del-Moral-Ávila R, et al. Effect of an Internet-based telehealth system on functional capacity and cognition in breast cancer survivors: a secondary analysis of a randomized controlled trial. Support Care Cancer. 2017;25(11):35519. DOI $10.1007 / \mathrm{s} 00520-017-3782-9$

13. Galiano-Castillo N, Cantarero-Villanueva I, Fernández-Lao C, Ariza-García A, Díaz-Rodríguez L, Del-Moral-Ávila R, et al. Telehealth system: a randomized controlled trial evaluating the impact of an internet-based exercise intervention on quality of life, pain, muscle strength, and fatigue in breast cancer survivors. Cancer. 2016;122(20):3166-74. DOI:https://doi.org/10.1002/cncr.30172

14. Kanera IM, Willems RA, Bolman CA, Mesters I, Verboon P, Lechner L. Long-term effects of a web-based cancer aftercare intervention on moderate physical activity and vegetable consumption among early cancer survivors: a randomized controlled trial. Int J Behav Nutr Phys Act. 2017;14(1):19. DOI 10.1186/s12966-017-0474-2

15. Kanera I, Bolman C, Willems R, Mesters I, Lechner L, Kanera IM, et al. Lifestylerelated effects of the web-based Kanker Nazorg Wijzer (Cancer Aftercare Guide) intervention for cancer survivors: a randomized controlled trial. J Cancer Surviv. 2016;10(5):883-97. DOI 10.1007/s11764-016-0535-6

16. Badger T, Segrin C, Dorros SM, Meek P, Lopez AM. Depression and Anxiety in Women with Breast Cancer and their Partners. Nurs Res. 2007;56(1):44-53.

17. Chung IY, Jung M, Park YR, Cho D, Chung H, Min YH, et al. Exercise Promotion and Distress Reduction Using a Mobile App-Based Community in Breast Cancer Survivors. Front Oncol. 2020;9:1505. DOI: https://doi.org/10.3389/fonc.2019.01505

18. Eakin EG, Lawler SP, Winkler EA, Hayes SC. A randomized trial of a telephonedelivered exercise intervention for non-urban dwelling women newly diagnosed 
with breast cancer: exercise for health. Ann Behav Med. 2012;43(2):229-38. DOI: $10.1007 / \mathrm{s} 12160-011-9324-7$

19. Frensham LJ, Parfitt G, Dollman J. Effect of a 12-Week Online Walking Intervention on Health and Quality of Life in Cancer Survivors: A QuasiRandomized Controlled Trial. Int J Environ Res Public Health. 2018;15(10). DOI: 10.3390/ijerph15102081

20. Garrett K, Okuyama S, Jones W, Barnes D, Tran Z, Spencer L, et al. Bridging the transition from cancer patient to survivor: Pilot study results of the Cancer Survivor Telephone Education and Personal Support (C-STEPS) program. Patient Educ Couns.

21. Quintiliani L, Mann DM, Puputti M, Quinn E, Bowen DJ. Pilot and Feasibility Test of a Mobile Health-Supported Behavioural Counselling Intervention for Weight Management Among Breast Cancer Survivors. JMIR Cancer. 2016;2(1):e4. doi: 10.2196/cancer.5305

22. Rocque GB, Halilova KI, Varley AL, Williams CP, Taylor RA, Masom DG, et al. Feasibility of a telehealth educational program on self-management of pain and fatigue in adult cancer patients. J Pain Symptom Manage. 2017;53(6):1071-8. DOI: http://dx.doi.org/10.1016/j.jpainsymman.2016.12.345

23. Short CE, Rebar A, James EL, Duncan MJ, Courneya KS, Plotnikoff RC, et al. How do different delivery schedules of tailored web-based physical activity advice for breast cancer survivors influence intervention use and efficacy? J Cancer Surviv. 2017;11(1):80-91. DOI 10.1007/s11764-016-0565-0

24. Bantum EO, Albright CL, White KK, Berenberg JL, Layi G, Ritter PL, et al. Surviving and thriving with cancer using a Web-based health behaviour change intervention: randomized controlled trial. J Med Internet Res. 2014;16(2):e54. DOI: 10.2196/jmir.3020

25. Ligibel JA, Partridge A, Giobbie-Hurder A, Campbell N, Shockro L, Salinardi T, et al. Physical and psychological outcomes among women in a telephone-based exercise intervention during adjuvant therapy for early stage breast cancer. $J$ Women's Health. 2010;19(8):1553-9. DOI: 10.1089=jwh.2009.1760

26. Nemli A, Tekinsoy Kartin P. Effects of exercise training and follow-up calls at home on physical activity and quality of life after a mastectomy. Jpn J Nurs Sci. 2019;16(3):322-8. doi:10.1111/jjns. 12243 
27. Uhm KE, Yoo JS, Chung SH, Lee JD, Lee I, Kim JI, et al. Effects of exercise intervention in breast cancer patients: is mobile health (mHealth) with pedometer more effective than conventional program using brochure? Breast Cancer Res Treat. 2017;161(3):443-52. DOI 10.1007/s10549-016-4065-8

28. Villaron C, Cury F, Eisinger F, Cappiello M-A, Marqueste T. Telehealth applied to physical activity during cancer treatment: a feasibility, acceptability, and randomized pilot study. Support Care Cancer. 2018;26(10):3413-21. DOI: https://doi.org/10.1007/s00520-018-4191-4

29. Bruns ERJ, Argillander TE, Schuijt HJ, van Duijvendijk P, van der Zaag ES, Wassenaar EB, et al. Fit4SurgeryTV At-home Prehabilitation for Frail Older Patients Planned for Colorectal Cancer Surgery: A Pilot Study. Am J Phys Med Rehab. 2019;98(5):399-406. DOI: 10.1097/PHM.0000000000001108

30. Cheong IY, An SY, Cha WC, Rha MY, Kim ST, Chang DK, et al. Efficacy of Mobile Health Care Application and Wearable Device in Improvement of Physical Performance in Colorectal Cancer Patients Undergoing Chemotherapy. Clin Colorectal Cancer. 2018;17(2):e353-e62. DOI: https://doi.org/10.1016/j.clcc.2018.02.002

31. Cheville AL, Moynihan T, Basford JR, Nyman JA, Tuma ML, Macken DA, et al. The rationale, design, and methods of a randomized, controlled trial to evaluate the effectiveness of collaborative telecare in preserving function among patients with late stage cancer and hematologic conditions. Contemp Clin Trials. 2018;64:25464. DOI: http://dx.doi.org/10.1016/j.cct.2017.08.021

32. Chung IY, Jung M, Lee SB, Lee JW, Park YR, Cho D, et al. An Assessment of Physical Activity Data Collected via a Smartphone App and a Smart Band in Breast Cancer Survivors: Observational Study. J Med Internet Res. 2019;21(9):13463. DOI: $10.2196 / 13463$

33. Cnossen IC, van Uden-Kraan CF, Rinkel RNPM, Aalders IJ, de Goede CJT, de Bree R, et al. Multimodal guided self-help exercise program to prevent speech, swallowing, and shoulder problems among head and neck cancer patients: A feasibility study. J Med Internet Res. 2014;16(3):282-93. DOI: 10.2196/jmir.2990

34. Gehring K, Kloek CJ, Aaronson NK, Janssen KW, Jones LW, Sitskoorn MM, et al. Feasibility of a home-based exercise intervention with remote guidance for patients with stable grade II and III gliomas: a pilot randomized controlled trial. Clin Rehabil. 2018;32(3):352-66. DOI: https://doi.org/10.1177/0269215517728326 
35. Haggerty AF, Hagemann A, Barnett M, Thornquist M, Neuhouser ML, Horowitz $\mathrm{N}$, et al. A randomized, controlled, multicentre study of technology-based weight loss interventions among endometrial cancer survivors. Obesity. 2017;25(Suppl 2):S102-S8. DOI:10.1002/oby.22021

36. Haggerty AF, Huepenbecker S, Sarwer DB, Spitzer J, Raggio G, Chu CS, et al. The use of novel technology-based weight loss interventions for obese women with endometrial hyperplasia and cancer. Gynec Oncol. 2016;140(2):239-44. https://doi.org/10.1016/j.ygyno.2015.11.033

37. Ji W, Kwon H, Lee S, Kim S, Hong JS, Park YR, et al. Mobile Health Management Platform-Based Pulmonary Rehabilitation for Patients With Non-Small Cell Lung Cancer: Prospective Clinical Trial. JMIR mHealth uHealth. 2019;7(6):e12645. doi: $10.2196 / 12645$

38. Mohamad H, Ntessalen M, Craig LCA, Clark J, Fielding S, N'Dow J, et al. A selfhelp diet and physical activity intervention with dietetic support for weight management in men treated for prostate cancer: pilot study of the Prostate Cancer Weight Management (PRO-MAN) randomised controlled trial. Br J Nutr. 2019;122(5):592-600. https://doi.org/10.1186/ISRCTN46025196.

39. Timmerman JG, Dekker-van Weering MGH, Stuiver MM, Groen WG, Wouters MWJM, Tönis TM, et al. Ambulant monitoring and web-accessible home-based exercise program during outpatient follow-up for resected lung cancer survivors: actual use and feasibility in clinical practice. J Cancer Surviv. 2017;11(6):720-31. 1 DOI 10.1007/s11764-017-0611-6

40. Vallerand JR, Rhodes RE, Walker GJ, Courneya KS. Social cognitive effects and mediators of a pilot telephone counselling intervention to increase aerobic exercise in hematologic cancer survivors. J Phys Act Health. 2019;16(1):43-51. DOI: https://doi.org/10.1123/jpah.2018-0014

41. Wosik J, Fudim M, Cameron B, Gellad ZF, Cho A, Phinney D, Curtis S, Roman M, Poon EG, Ferranti J, Katz JN. Telehealth Transformation: COVID-19 and the rise of Virtual Care. Journal of the American Medical Informatics Association. 2020 Jun 1;27(6):957-62. DOI: https://doi.org/10.1093/jamia/ocaa067

42. Royce TJ, Sanoff HK, Rewari A. Telemedicine for Cancer Care in the Time of COVID-19. JAMA Oncol. 2020 Jul 16. DOI: doi:10.1001/jamaoncol.2020.2684 
43. Elkaddoum R, Haddad FG, Eid R, Kourie HR. Telemedicine for cancer patients during COVID-19 pandemic: between threats and opportunities. Future Oncol. 2020 May 01;16(18)DOI: https://doi.org/10.2217/fon-2020-0324

44. Wang Y, Duan Z, Ma Z, Mao Y, Li X, Wilson A, Qin H, Ou J, Peng K, Zhou F, Li C. Epidemiology of mental health problems among patients with cancer during COVID-19 pandemic. Trans Psych. 2020 Jul 31;10(1):1-0. Available from: https://www.nature.com/articles/s41398-020-00950-y

45. Kinney AY, Boonyasiriwat W, Walters ST, Pappas LM, Stroup AM, Schwartz MD, Edwards SL, Rogers A, Kohlmann WK, Boucher KM, Vernon SW. Telehealth personalized cancer risk communication to motivate colonoscopy in relatives of patients with colorectal cancer: the family CARE Randomized controlled trial. $J$ Clinic Oncol. 2014 Mar 1;32(7):654. DOI: 10.1200/JCO.2013.51.6765

46. Worster, Brooke, and Kristine Swartz. "Telemedicine and palliative care: an increasing role in supportive oncology." Curr Oncology Report. 2017 Jun 1;19(6):37. DOI: 10.1007/s11912-017-0600-y 